\title{
Monte Carlo Studies of medium-size telescope designs for the Cherenkov Telescope Array
}

\author{
M.D. Wood ${ }^{\mathrm{a}}$, T. Jogler ${ }^{\mathrm{a}}$, J. Dumm $^{\mathrm{b}}$, S. Funk ${ }^{\mathrm{a}}$ \\ ${ }^{a}$ SLAC National Accelerator Laboratory, 2575 Sand Hill Road M/S 29, Menlo Park, CA 94025, USA \\ ${ }^{b}$ Oskar Klein Centre and Dept. of Physics, Stockholm University, SE-10691 Stockholm, Sweden
}

\begin{abstract}
We present studies for optimizing the next generation of ground-based imaging atmospheric Cherenkov telescopes (IACTs). Results focus on mid-sized telescopes (MSTs) for CTA, detecting very high energy gamma rays in the energy range from a few hundred $\mathrm{GeV}$ to a few tens of $\mathrm{TeV}$. We describe a novel, flexible detector Monte Carlo package, FAST (FAst Simulation for imaging air cherenkov Telescopes), that we used to simulate different array and telescope designs. The simulation is somewhat simplified to allow for efficient exploration over a large telescope design parameter space. We studied a wide range of telescope performance parameters including optical resolution, camera pixel size, and light collection area. In order to ensure a comparison of the arrays at their maximum sensitivity, the simulations were analyzed with the most sensitive techniques used in the field, such as maximum likelihood template reconstruction and boosted decision trees for background rejection. Choosing telescope design parameters representative of the proposed Davies-Cotton (DC) and Schwarzchild-Couder (SC) MST designs, we compared the performance of the arrays. In particular, we examined the gamma-ray angular resolution and differential point-source sensitivity under a wide range of conditions, determining the impact of the number of telescopes, telescope separation, night sky background, and geomagnetic field. We found a 30-40\% improvement in the gamma-ray angular resolution at all energies when comparing arrays with an equal number of SC and DC telescopes, significantly enhancing pointsource sensitivity in the MST energy range. The increase in point-source sensitivity can be attributed to the improved optical point-spread function and smaller pixel size.
\end{abstract}

Keywords: Monte Carlo simulations, Cherenkov telescopes, IACT technique, gamma rays, cosmic rays

\section{Introduction}

The ground-based imaging atmospheric Cherenkov telescope (IACT) technique has led to significant progress in the field of very high energy (VHE; $E>$ $100 \mathrm{GeV}$ ) gamma-ray astronomy over the last 25 years. To date, 145 sources have been detected at VHE with $\sim 60$ sources discovered only in the last five years ${ }^{11}$ IACTs allow us to study a wide range of scientific topics, many uniquely accessible by VHE astronomy. Current and future generations of IACTs aim to probe the origins and acceleration processes of cosmic rays [1, 2,

\footnotetext{
${ }^{*}$ Corresponding author

Email addresses: mdwood@slac.stanford.edu (M.D. Wood) tjogler@slac.stanford.edu (T. Jogler), jon.dumm@fysik.su.se (J. Dumm), funk@slac.stanford.edu (S. Funk)

${ }^{1}$ http://tevcat.uchicago.edu/
}

Preprint submitted to Astroparticle Physics
3] and explore the nature of black holes and their relativistic jets. Other key objectives include the search for dark matter, axion-like particles [4, 5], and Lorentz invariance violation [6]. This will require extensive observations on a number of source classes such as pulsars and pulsar wind nebulae [7], galactic binaries [8], supernova remnants [9], active galactic nuclei [10, 11], and gamma-ray bursts [12, 13]. The extragalactic sources can be used as "backlights" to study the attenuation on the extragalactic background light, useful for constraining star formation history and other cosmological parameters such as the Hubble constant [14].

VHE gamma rays entering the Earth's atmosphere undergo $e^{+} e^{-}$pair production, initiating electromagnetic cascades. The relativistic charged particles in the shower emit Cherenkov ultraviolet and optical radiation, which is detectable at ground level. The majority of the emitted Cherenkov light is narrowly beamed along the trajectory of the gamma-ray primary in a cone

March 27, 2015 
with an opening angle of $\sim 1.0^{\circ}$. Due to the beaming effect, the majority of the Cherenkov light falls within 84 a Cherenkov light pool with a diameter of 200-300 m and a nearly constant light density. By imaging the Cherenkov light emitted by the shower particles, IACTs are able to reconstruct the direction and energy of the original gamma ray and to distinguish gamma rays from the much more prevalent cosmic-ray background. High resolution imaging of the Cherenkov shower offers significant benefits for IACTs by enabling a more accurate measurement of the shower axis which has an intrinsic transverse angular size of only a few arcminutes. However the finite shower width and stochastic fluctuations in the shower development fundamentally limit the performance of IACTs.

The designs of IACTs are governed by a few key factors. At low energy, the number of Cherenkov photons compared to the night sky background necessitates a 100 large $O(10-20 \mathrm{~m})$ mirror diameter and high quantum 101 efficiency camera. The camera must also be able to 102 capture the signal very quickly since the duration of 103 a Cherenkov pulse can be as short as a few nanosec- 104 onds. The optical point-spread function (PSF) and cam- 105 era pixel size should ideally be suitably smaller than 106 the angular dimension of the gamma-ray shower. How- 107 ever the high cost-per-pixel of camera designs used in 108 current generation IACTs has generally dictated pixel 109 sizes that are significantly larger $\left(0.1^{\circ}-0.2^{\circ}\right)$ than the 110 angular size of shower structure. Multiple viewing an- 111 gles of the same shower offered by an array of tele- 112 scopes drastically improves the reconstruction perfor- ${ }_{113}$ mance and background rejection. Finally, at high en- 114 ergy, the sensitivity of IACTs is limited by gamma-ray 115 signal statistics, requiring an array with a large effective ${ }_{116}$ gamma-ray collection area.

The current generation of IACTs all have single-dish 118 optical systems. These have small spherical mirror 119 facets attached to either a spherical dish (i.e. Davies- 120 Cotton (DC) [15, 16]) or a parabolic dish. The parabolic ${ }_{121}$ dish reduces the time spread of the Cherenkov signal 122 but introduces a larger off-axis optical PSF. An interme- 123 diate design with a spherical dish but a larger radius of 124 curvature (intermediate-DC) can be used to achieve an 125 improved time spread while maintaining off-axis perfor- 126 mance [17, 18]. These single-dish designs are appealing ${ }_{127}$ because they are relatively inexpensive, mirror align- 128 ment is straightforward, and the optical PSF at large ${ }_{129}$ field angles is better than that of monolithic spherical 130 or parabolic reflectors [19].

The possibility of improving the PSF (especially off axis) and reducing the plate scale of IACTs has driven the study of Schwarzschild-Couder (SC) apla- natic telescopes with two aspheric mirror surfaces ${ }^{2}$. The improved PSF across the field of view (FoV) allows for more accurate surveying and mapping of extended sources. The reduced plate scale is highly compatible with new camera technologies such as Silicon photomultipliers or multi-anode photomultiplier tubes. These technologies allow for a cost-effective, finely-pixelated image over a large FoV. Studies have been performed providing solutions for mirror surfaces optimized to correct spherical and coma aberrations. These solutions are also isochronous, allowing for a short trigger coincidence window [20]. The first SC prototype is still being developed [21] and has several challenges to overcome. In particular, the tolerances of the mechanical structure in the camera and mirror alignment system are relatively stringent, which translates to a higher cost. To provide comparisons at a fixed cost, our SC simulations use a smaller mirror area than that of the baseline DC design.

The Cherenkov Telescope Array (CTA) is an example of a next-generation IACT observatory. CTA aims to surpass the current IACT systems such as H.E.S.S. [22], MAGIC [23] and VERITAS [24] by an order of magnitude in sensitivity and enlarge the observable energy range from a few tens of $\mathrm{GeV}$ to beyond one hundred $\mathrm{TeV}$ [25]. To achieve this broad energy range and high sensitivity, CTA will incorporate telescopes of three different sizes spread out over an area of $\sim 3 \mathrm{~km}^{2}$. Telescopes are denoted by their mirror diameter as largesize telescopes (LSTs, $\sim 24 \mathrm{~m}$ ), medium-size telescopes (MSTs, $\sim 12 \mathrm{~m}$ ), and small-size telescopes (SSTs, $\sim 4 \mathrm{~m}$ in the current design). The baseline designs for the LST and MST both feature a single reflector based on the DC optical design. Telescope designs based on dualreflector SC optics are also being developed for both medium- and small-sized telescopes. The medium-size SC telescope (SCT) would fill a similar role to the MST and predominantly contribute to the sensitivity of CTA in the energy range between $100 \mathrm{GeV}$ and $1 \mathrm{TeV}$. In this paper we explore a range of telescope models but focus primarly on the comparison of designs with characteristics similar to the MST and SCT. In the subsequent discussion we use MST to refer to all telescope designs with a primary mirror diameter of 9-12 m. DC-MST and SC-MST are used to specifically refer to telescopes with the imaging characteristics similar to the MST and SCT designs, respectively.

The baseline design of CTA includes $\sim$ four LSTs, $\sim 30$ MSTs, and $\sim 50$ SSTs. The sensitivity could be improved by a factor of $2-3$ in the core energy range

\footnotetext{
${ }^{2}$ Though segmented, the mirror surfaces are often referred to as a singular mirror for brevity.
} 
by expanding the MST array with an additional 24-36 180 SCTs. With these additional telescopes, the combined ${ }_{18}$ MST and SCT array enters a new regime where the in- 182 ternal effective area is comparable to the effective area ${ }_{183}$ of events landing outside the array. These so-called con- 184 tained events have much improved angular and energy resolution as well as background rejection. Extensive ${ }_{185}$ work is underway to optimize the design of CTA for the wide range of science goals [18]. The scope of previous studies has been primarily on a straightforward expansion of existing telescope designs to larger arrays.

In this paper, we describe a novel, flexible Monte Carlo simulation and analysis chain. We use them to evaluate the performance of CTA-like arrays over a large range of telescope configurations and design parameters. Section 2 describes this simulation and the simplified detector model. In Section 3, we explain the analysis chain, including a maximum likelihood shower reconstruction using simulated templates. This reconstruction was used for comparisons between the maximum sensitivity for each array configuration. In Section 4, we show comparisons between possible CTA designs, focusing primarily on the number of telescopes and the DC versus SC designs. We conclude in Section 5.

\section{Simulation}

We have studied the performance of a variety of array 206 geometries and telescope configurations for a hypothet- 207 ical CTA site at an altitude of $2000 \mathrm{~m}$. Details of the ${ }_{208}$ site model and array geometry are described in Sections 209 2.1 and 2.2. Simulations of the telescope response were 210 performed using a simplified detector model described ${ }_{211}$ in Section 2.3.

\subsection{Air-Shower Simulations}

Simulations of the gamma-ray and cosmic-ray air shower cascades were performed with the CORSIKA v6.99 Monte Carlo (MC) package [26] and the QGSJet II-03 hadronic interaction model [27]. We used a site model with an elevation of $2000 \mathrm{~m}$, a tropical atmospheric profile, and an equatorial geomagnetic field configuration with $\left(B_{x}, B_{z}\right)=(27.5 \mu \mathrm{T},-15.0 \mu \mathrm{T})$. This site model is identical to the one used in [18] and has similar characteristics to the southern hemisphere sites proposed for CTA.

Gamma-ray showers were simulated as coming from ${ }^{224}$ a point on the sky at $20^{\circ}$ zenith angle and $0^{\circ}$ azimuth 225 angle, as measured from the local magnetic north over 226 the energy range from $10 \mathrm{GeV}$ to $30 \mathrm{TeV}$. Protons and ${ }_{227}$ electrons were simulated with an isotropic distribution 228 that extends to $8^{\circ}$ and $5^{\circ}$ respectively from the direction of the gamma-ray primary. We use the spectral parameterizations for proton and electron fluxes from [28]. To account for the contribution of heavier cosmic-ray nuclei we increase the proton flux by a factor 1.2.

\subsection{Array Geometry}

Proposed designs for CTA employ three telescope types (SST, MST, and LST) with variable intertelescope spacing from $120 \mathrm{~m}$ to more than $200 \mathrm{~m}$ [18]. The number of telescopes of each type and their separations are chosen to optimize the differential sensitivity over the full energy range of CTA. [18] found that two balanced arrays (arrays E and I) that have 3-4 LSTs, 18-23 MSTs, and 30-50 SSTs of $7 \mathrm{~m}$ diameter provide the best compromise in performance over the full energy range of CTA while keeping the total cost of the array within the projected CTA budget.

For this study we simulated an array geometry which is similar to the one used for MSTs and LSTs in arrays $\mathrm{E}$ and I. The array is composed of 61 telescopes arranged on a grid with constant inter-telescope spacing of $120 \mathrm{~m}$ (see Figure 1). A telescope spacing of about $120 \mathrm{~m}$ is well motivated by the characteristic size of the Cherenkov light pool for gamma-ray air showers and guarantees that multiple telescopes will sample the shower within the shower light pool. Subsets of telescopes from the baseline array were used to construct arrays with a reduced number of telescopes by removing successive rings of telescopes along the array perimeter. These reduced arrays have between 5 and 41 telescopes and encompass arrays that are similar in telescope number to both current IACT arrays $\left(\mathrm{N}_{\text {tel }}=\right.$ 5 ) and the array designs currently considered for CTA $\left(\mathrm{N}_{\text {tel }}=25-41\right)$. We also examined the performance of arrays with smaller and larger inter-telescope separations $(60 \mathrm{~m}-200 \mathrm{~m})$ by rescaling the inter-telescope separation of our baseline array.

All simulations were performed with homogeneous arrays composed of a single telescope type. We primarily consider telescope models with mirror areas between the current MST and LST designs. Because our study is focused on the performance of arrays in the core CTA energy range $(100 \mathrm{GeV}-10 \mathrm{TeV})$ we did not consider SSTs.

\subsection{Detector Model}

Simulations of IACT arrays have traditionally been performed with highly detailed detector models that use optical ray-tracing to track the trajectory and time of arrival of individual Cherenkov photons. Because these 


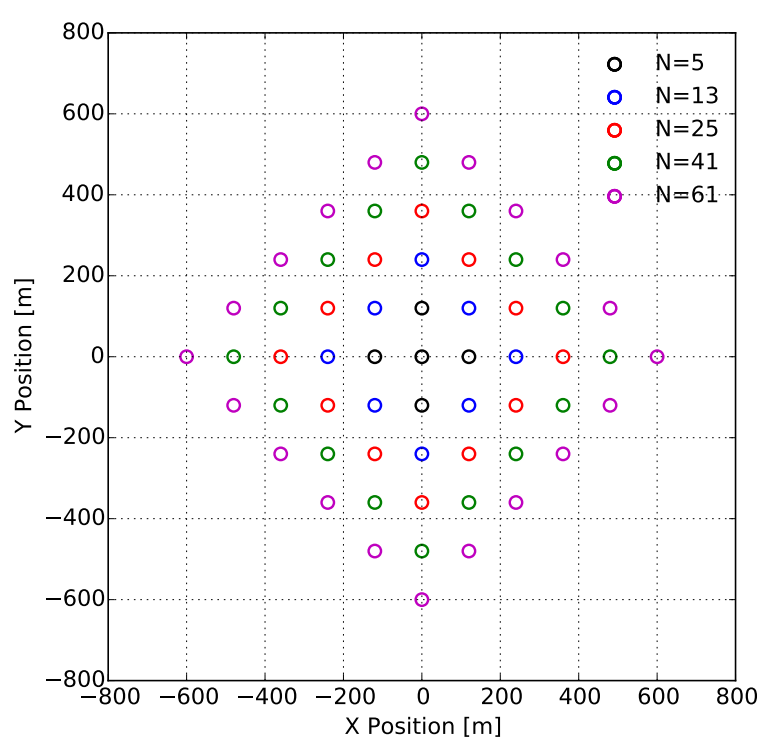

Figure 1: Physical telescope positions for the five array geometries 268 used for this study. All geometries are composed of telescopes ar- 269 ranged on a uniform grid with $120 \mathrm{~m}$ spacing. The smallest array 270 is composed of five telescopes (black circles). The larger arrays are constructed by the addition of successive rings of telescopes around the array boundary up to a maximum of 61 telescopes in the baseline 272 array geometry. significant computational challenge. In order to effi-
ciently study the telescope design parameter space, we have developed a simplified telescope simulation tool, FAST (FAst Simulation for imaging air cherenkov Telescopes), that is not tied to any particular mirror configuration or camera technology. In the FAST model, the telescope characteristics are fully described by the following parameters:

\section{- Effective light collection area: $A_{\mathrm{opt}}$}

- $68 \%$ containment radius of the optical PSF: $R_{\mathrm{psf}}$

- Camera pixel size: $D_{\text {pix }}$

- Effective camera trigger threshold: $T_{\text {th }}$

- Single photo-electron (PE) charge resolution: $\sigma_{\mathrm{spe}}{ }^{286}$

- Pixel read-noise: $\sigma_{b}$

- Effective integration window: $\Delta T$
8 70 70 271 272

$$
\text { of }
$$

where

is the normalized wavelength distribution of Cherenkov light at the ground for an emission altitude $z$ and an optical depth for atmospheric extinction $\tau(\lambda, z)$. We use an atmospheric extinction model generated with MODTRAN [29] for the tropical atmosphere and an aerosol layer with a visibility of $50 \mathrm{~km}$. For all further evaluations of $A_{\text {opt }}$ we use $z=10 \mathrm{~km}$ and an integration over wavelength from $250 \mathrm{~nm}$ to $700 \mathrm{~nm}$.

We define a benchmark telescope with a $D=12 \mathrm{~m}$ primary diameter and a total photon detection efficiency that includes losses from mirror reflections and photosensor efficiency. We use a photosensor model with a spectral response that is characteristic of photomultiplier tubes and has a peak efficiency of $24 \%$ at $350 \mathrm{~nm}$. Losses from mirror reflections are evaluated for a single optical surface using a wavelength-dependent reflectivity with a peak efficiency of $89 \%$ at $320 \mathrm{~nm}$. This reflectivity is similar to that of the aluminum and aluminized glass mirrors used in current generation IACTs. Figure 2 shows the optical effective area of the telescope model 


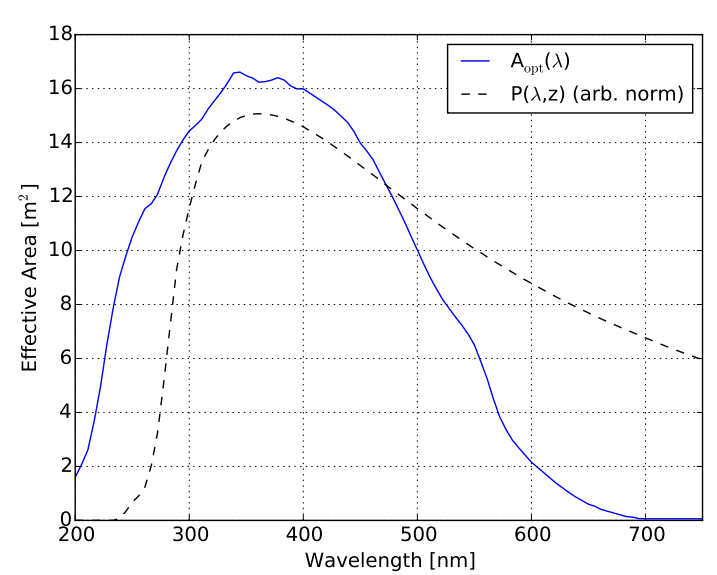

Figure 2: Effective light collection area versus wavelength for the benchmark telescope model with $A_{\text {opt }}=11.18 \mathrm{~m}^{2}$. The dashed black line shows the spectral shape for Cherenkov light emitted at an eleva- 33 tion of $10 \mathrm{~km}$ after absorption by the atmosphere.

as a function of wavelength. The effective light collection area of our benchmark telescope is $11.18 \mathrm{~m}^{2}$ which is representative of medium-sized IACTs with $\sim 10 \mathrm{~m}$ aperture and $50-100 \mathrm{~m}^{2}$ mirror area. The response of telescopes with larger or smaller light collection areas is modeled using the same spectral response and mirror area as the benchmark telescope model but scaling the photon detection efficiency by the ratio $A_{\mathrm{opt}} / 11.18 \mathrm{~m}^{2}$.

The imaging response of the telescope optical system is simulated by applying a model for the optical pointspread-function (PSF) to the distribution of true photon arrival directions in the camera image plane. After applying a survival probability for detection, each Cherenkov photon is assigned a random offset drawn from the optical PSF. We parameterize the optical PSF as a $2 \mathrm{D}$ gaussian with a $68 \%$ containment radius, $R_{\mathrm{psf}},{ }_{358}$ that is constant across the FoV. We consider values of 359 $R_{\text {psf }}$ between $0.02^{\circ}$ and $0.08^{\circ}$ which is comparable to 360 the range of PSF spot sizes for the CTA telescope designs at both small and large field angles. All telescopes are simulated with an 8 deg FoV with a light collection area that is constant with field angle.

Telescopes are simulated with a camera geometry 365 composed of square pixels of angular width $D_{\text {pix }}$ that ${ }_{366}$ uniformly tile the camera FoV. Each pixel is assigned ${ }_{367}$ a time integrated signal that is the sum of the detected ${ }_{368}$ Cherenkov photons, night-sky background (NSB) pho- ${ }_{369}$ tons, and detector noise. The number of NSB photons 370 is drawn from a Poisson distribution where the average 371 $\left(\mu_{b}\right)$ is computed using an implicit time integration win- 372 dow $(\Delta T)$ of $16 \mathrm{~ns}$. The mean number of NSB photons ${ }_{373}$$$
339
$$

\section{.}



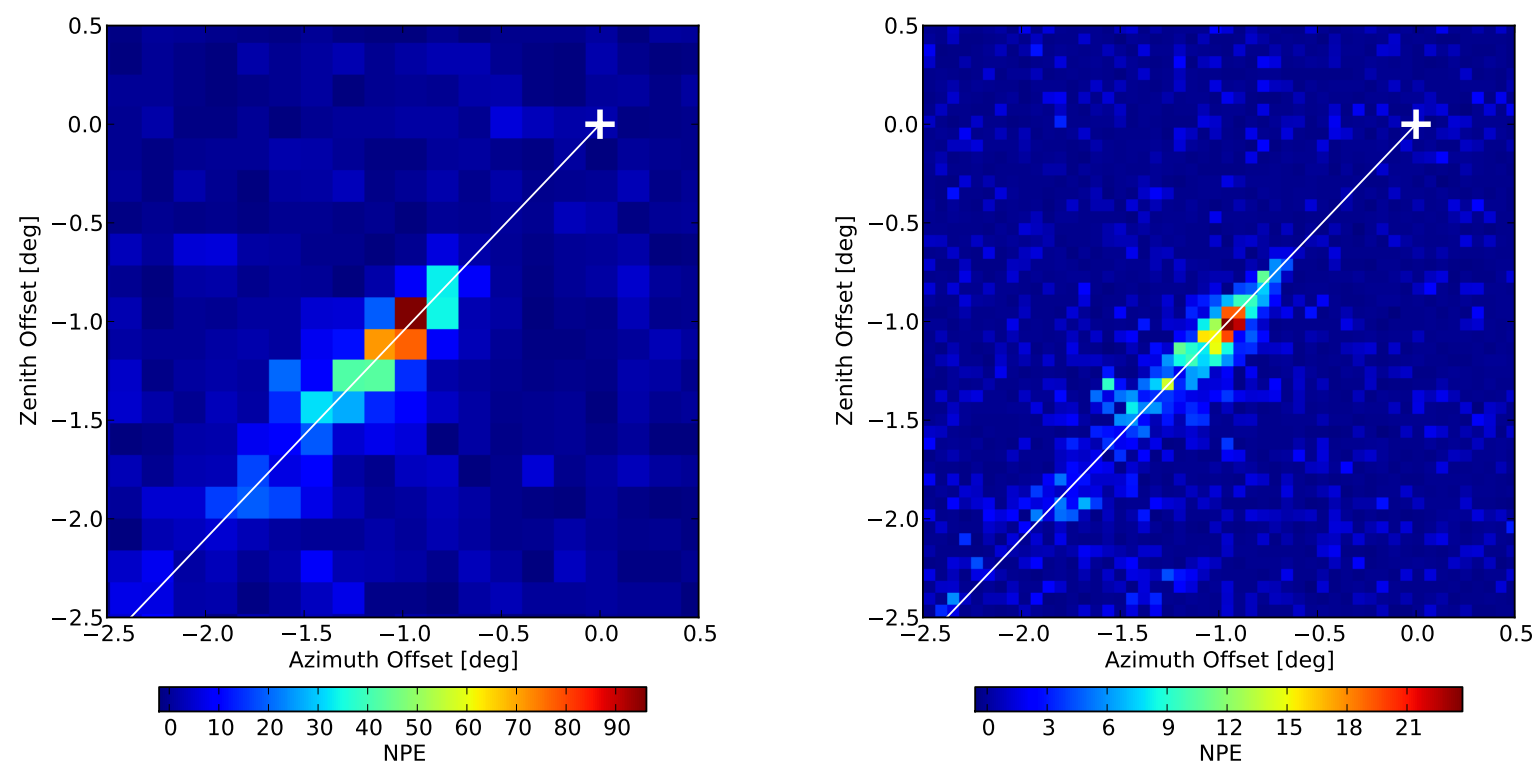

Figure 3: Camera images of the same $1 \mathrm{TeV}$ gamma-ray shower with an impact distance of $120 \mathrm{~m}$ simulated with two different telescope pixel sizes: $D_{\text {pix }}=0.16^{\circ}$ (left) and $D_{\text {pix }}=0.06^{\circ}$ (right). Both telescope models have $A_{\text {opt }}=11.18 \mathrm{~m}^{2}$ and $R_{\text {psf }}=0.02^{\circ}$. The color scale denotes the measured signal amplitude in PEs for each pixel. The white cross and solid line show the direction of the gamma-ray primary and the projection of its trajectory to the telescope image plane, respectively.

telescopes, the array-level trigger is simulated requiring 400 a multiplicity of at least two triggered telescopes. The 401 camera threshold provides a single parameter model that 402 we use to explore influence of the trigger threshold on 403 the array-level performance. By calibrating $T_{\text {th }}$ to the 404 effective camera threshold of a given trigger design, we can also approximate the trigger response that would be obtained with a more detailed trigger simulation implementation.

Studies performed with the sim telarray detector 405 simulation package [28] have shown that camera trigger ${ }_{407}$ designs currently considered for the MSTs can achieve ${ }_{408}$ effective trigger thresholds of 60-80 PE for a single tele- ${ }_{409}$ scope accidental trigger rate of $1-10 \mathrm{kHz}$. We adopted ${ }_{410}$ a trigger threshold of $60 \mathrm{PE}$ for our baseline telescope ${ }_{411}$ model with $A_{\text {opt }}=11.18 \mathrm{~m}^{2}$ which is comparable to the ${ }_{412}$ effective threshold of the prod-2 MST model [33]. To ${ }_{413}$ model the effective trigger threshold for telescopes with different light collection areas, we used a simple scaling formula that approximates the threshold needed to maintain a constant rate of accidental triggers. If the to- 415 tal pixel noise is dominated by NSB photons, the rate of ${ }_{416}$ accidental triggers should be proportional to the RMS 417 fluctuations in the number of NSB photons collected in 418 a trigger pixel which scales as $A_{\mathrm{opt}}^{1 / 2}$ if the angular pixel ${ }_{419}$ size is held fixed. Telescopes with larger effective light 420 collection area achieve a lower trigger threshold through the suppression of these NSB fluctuations relative to the signal amplitude which increases linearly with $A_{\text {opt }}$. We assign the effective trigger threshold for a telescope with light collection area $A_{\text {opt }}$ as,

$$
T_{\text {th }}=60 \mathrm{PE}\left(\frac{A_{\mathrm{opt}}}{11.18 \mathrm{~m}^{2}}\right)^{1 / 2} .
$$

For the studies presented in Section 4, we consider a benchmark array (M61) with 61 identical telescopes with $A_{\mathrm{opt}}=11.18 \mathrm{~m}^{2}, R_{\mathrm{psf}}=0.02^{\circ}, D_{\mathrm{pix}}=0.06^{\circ}$, and $T_{\mathrm{th}}=60 \mathrm{PE}$. Our baseline telescope model is representative of a generic medium-sized telescope design with SC-like imaging characteristics. In Section 4.2 we additionally consider other telescope models that were specifically chosen to match the characteristics of the proposed CTA telescope designs.

\subsection{Simplifications of the Detector Simulation}

The FAST package uses a highly simplified model of the telescope optics and camera. This allows us to perform a more general exploration of the IACT design parameter space without focusing on the details of any specific optical design or camera technology. These simplifications also make the FAST simulation much 
less computationally intensive than traditional simula- 473 tion tools such as sim_telarray which further facili- 474 tates the exploration of a large phase space of telescope 475 and array design concepts. When simulating compa- 476 rable telescope designs with FAST and sim_telarray 477 we observe an order-of-magnitude reduction in compu- 478 tation time. Here, we discuss the limitations of the ap- 479 proach taken in the FAST simulation package and de- 480 scribe the areas in which a more detailed simulation of 481 the telescope camera and optics could potentially affect ${ }_{482}$ our results.

FAST does not use raytracing to account for shadow- 484 ing of the camera by telescope structure and assumes a 485 simple 2D gaussian PSF that is constant over the FoV. ${ }_{486}$ Full raytracing simulations can be used to model effects 487 such as shadowing by the telescope structure and light 488 losses from gaps in the mirror surfaces. Raytracing also ${ }_{489}$ allows a more realistic modeling of the telescope PSF. 490 Optical aberrations intrinsic to the design of IACTs in- 491 troduce a strong field-angle dependence to both the size 492 and shape of the PSF. In the FAST telescope model, all ${ }_{493}$ effects that influence the optical performance of the tele- 494 scope are folded into the effective optical area $\left(A_{\text {opt }}\right)$ and 495 the $68 \%$ containment radius of the PSF $\left(R_{\mathrm{psf}}\right)$. Shadow- 496 ing and other light losses in the optical system are thus 497 taken into account by a reduction in the effective optical area. The field-angle dependence of the PSF is studied by comparing the performance of telescope designs with the best and worst PSF at any field angle. The performance of a real telescope should always fall between that of telescopes with smallest and largest PSF in the FoV. We did not study the effect of an asymmetric PSF but it is plausible to assume that the array performance with telescopes that have an asymmetric PSF can be estimated by enlarging the symmetric PSF in FAST.

FAST does not simulate the timing of signals and assumes an ideal data acquisition system that is only lim- ${ }^{505}$ ited by the irreducible noise from NSB photons. Effects 506 that distort the measurement of the pixel charge such 507 as cross-talk, after-pulsing, timing jitter, non-linearity, 508 and saturation might lead to a reduction in performance. 509 However these effects should affect all telescope types 510 in a similar way in the sense that photon charges might 511 be only partially reconstructed. Per the telescope perfor- 512 mance requirements set forward by CTA, we expect the 513 influence of these effects to be sub-dominant to the irre- 514 ducible limitations on the IACT technique set by shower 515 physics (NSB and shower fluctuations). Many of these 516 effects can be partially mitigated with pixel-level cali- 517 bration or pre-processing analysis procedures. For in- 518 stance, suppression algorithms such as clipping of pixel 519 signal amplitudes or the removal of isolated high PE 520 pixels have been demonstrated as efficient techniques to reduce the impact of after-pulsing. In principle these effects could also be approximated in the FAST detector model by increasing the pixel-level noise or worsening the charge resolution. We note that our analysis does not use shower timing parameters or shower development timing in any explicit way and thus is robust with respect to changes in timing requirements.

Another simplification in FAST is the trigger threshold decision logic that assumes any shower image above a certain threshold will trigger the telescope. Realistic trigger electronics might have several characteristics that have to be simulated in detail but in the early planning state of an array our method is very valuable to give a solid estimate of the array performance as long as the trigger threshold is not chosen aggressively and the requirements of the telescope array are met. Indeed we show in Section 4.3 that our telescope threshold might be too conservative given that more realistic simulations find enhanced performance near the energy threshold of simulated arrays. For relative comparisons of arrays our simplifications are unimportant. To estimate the influence on the absolute performance impacts of our simplified simulations we compare our results to a much more sophisticated detector simulation in Section 4.3 .

\section{Analysis}

The analysis of the telescope image data is performed using well established techniques for the analysis of IACT data. The analysis is performed in three stages: preparation of the telescope images, reconstruction of the event properties, and training and optimization of cuts.

\subsection{Image Cleaning and Parameterization}

The image analysis is applied to the telescope pixel amplitudes to derive a set of telescope-level parameters which characterize the distribution of light in each telescope. Analysis of the telescope image data begins with the application of an image cleaning analysis that selects pixels that have a signal amplitude that is larger than noise. Traditionally image cleaning has been performed using variations of a nearest-neighbor algorithm [34]. A search is performed for groups of neighboring pixels which exceed a threshold defined in terms of the absolute amplitude or the amplitude relative to the RMS noise in the pixel. These algorithms work well as long as the dimension of the pixel is of the same order as the Cherenkov image size. However in the limit of small pixel sizes these algorithms will lose efficiency for low 
energy showers where the signal is spread out over too many pixels to be discernible above noise when only considering nearest neighbors.

In order to circumvent the limitations of the nearestneighbor pixel algorithms, we use an Aperture cleaning algorithm that performs a smoothing over the camera with an angular scale $\left(R=0.12^{\circ}\right)$ that is of the same order as the width of a gamma-ray induced Cherenkov shower $\left(0.1-0.2^{\circ}\right)$.

In order to detect efficiently images that lie on pixel boundaries we divide each pixel into $N \times N$ subpixels where $N=\left\lceil D_{\text {pix }} / 0.06^{\circ}\right\rceil$. We compute the image intensity in the neighborhood of subpixel $i$ as

$$
\bar{s}(R)=\sum_{j} s_{j} w_{i, j}(R),
$$

where $w_{i, j}(R)$ is the fraction of the solid angle of pixel $j$ contained within the circular aperture of radius $R$ centered on subpixel $i$ (see Figure 4). The pixel image threshold is defined relative to the expected noise within the pixel aperture

$$
\sigma(R)=\left(\sum_{j}\left(\sigma_{b}^{2}+\mu_{b}\right) w_{i, j}(R)\right)^{1 / 2} .
$$

For the present analysis we adopt an image threshold ${ }_{570}$ of $\bar{s} / \sigma=7$ which for our baseline array corresponds 571 to an image intensity of $319 \mathrm{PE} \mathrm{deg^{-2 }}$ and an inte- ${ }_{572}$ grated charge of 14.4 PE within the cleaning aperture. 573 Any pixel for which one or more subpixels exceeds the 574 cleaning threshold is flagged as an image pixel. The 575 simulations do not include photodetector after-pulsing, 576 which can cause noise isolated in single pixels. These 577 may need to be suppressed if the aperture cleaning 578 method is applied in other scenarios. Telescope images 579 are discarded at this point if fewer than three image pix- 580 els are present.

The image cleaning is only used by the geometric re- 582 construction, itself a seed for the likelihood reconstruc- 583 tion. As such, a relatively low threshold was chosen to 584 maximize the reconstruction efficiency for low-energy 585 events.

Following the image cleaning analysis, an image 587 analysis is applied to the amplitudes of image pixels $\left(s_{j}\right) 588$ to calculate a set of image parameters that characterize 589 the light distribution in the focal plane. The image pa- 590 rameters include the total image size, $S$, the image cen- 591 troid, the second central moments along the major and 592 minor axes of the image denoted as length $l$ and width ${ }_{593}$ $w$, and the orientation of the major axis in the image 594 plane.
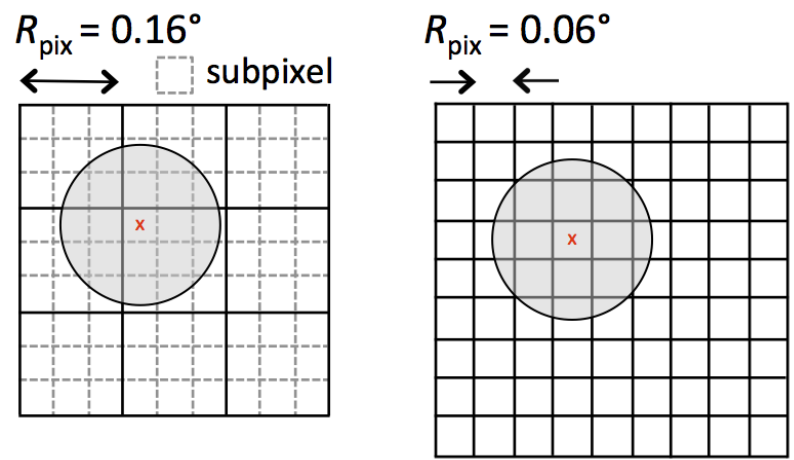

Figure 4: Illustration of the aperture cleaning algorithm on small camera subsections with $R=0.12^{\circ}$. In the DC-like case (left), pixels are subdivided since they are large compared to the aperture. Each subpixel is used as the center of an aperture for image intensity calculation. This calculation is based on the number of PEs and the fraction of the pixel area within the aperture, normalized to the area of the aperture. For the SC-like case (right), smaller pixels do not require subdivision.

\subsection{Shower Reconstruction}

The shower reconstruction determines a trajectory and energy for each event by fitting a shower model to the telescope image data. The shower model parameters $(\boldsymbol{\theta})$ are the primary energy $(E)$, the primary direction $(\mathbf{e})$, the primary impact position $(\mathbf{R})$, and the atmospheric column depth of the first interaction point $(\lambda)$. In an array of IACTs, each telescope views the shower from a different perspective and provides an independent constraint on the shower parameters. By using image data from multiple telescopes, one can perform a stereoscopic reconstruction of the shower trajectory. For the analysis algorithms presented in this section, we assume on-axis observations of a gamma-ray source in parallel pointing mode whereby the optical axes of the telescopes in the array are aligned with the shower direction. However the procedures described here can be also applied to the case of non-aligned telescope pointing.

In presenting the implementation of the shower reconstruction algorithms, we use a global coordinate system defined with the $x$-axis parallel to the direction of magnetic north and the $z$-axis perpendicular to the Earth's surface. The positions of the array telescopes are denoted by $\mathbf{r}_{i}$. For the array layouts considered for this study, the telescopes are arranged in a regular grid in the $x-y$ plane with all telescopes located at the same height above sea level $(z=2000 \mathrm{~m})$. Shower reconstruction is performed in a shower coordinate system with the $z$-axis aligned with the shower trajectory and defined by the basis vectors: 


$$
\begin{aligned}
& \hat{z}^{\prime}=\mathbf{e} \\
& \hat{y}^{\prime}=\frac{(\mathbf{e}-(\hat{z} \cdot \mathbf{e}) \hat{z})}{\sqrt{1-(\hat{z} \cdot \mathbf{e})^{2}}} \times \hat{z} \\
& \hat{x}^{\prime}=\hat{z}^{\prime} \times \hat{y}^{\prime}
\end{aligned}
$$

We use $\mathbf{r}_{i}^{\prime}$ and $\mathbf{R}^{\prime}$ to represent the projections of the telescope positions and the shower impact position to the $x^{\prime}-y^{\prime}$ plane. An illustration of the geometry of a shower is shown in Figure 5 The shower impact vector,

$$
\boldsymbol{\rho}_{i}=\mathbf{R}-\mathbf{r}_{i}-\left(\hat{z}^{\prime} \cdot\left(\mathbf{R}-\mathbf{r}_{i}\right)\right) \hat{z}^{\prime},
$$

describes the location of the shower impact position relative to telescope $i$ in the $x^{\prime}-y^{\prime}$ plane. The shower impact distance $\left(\rho_{i}=\left|\boldsymbol{\rho}_{i}\right|\right)$ is the distance of closest approach between the shower and the telescope.

The geomagnetic field (GF) alters the development of the gamma-ray shower by deflecting the charged particles in the electromagnetic cascade. The GF deflects particles in a plane perpendicular to their trajectories with a strength proportional to the perpendicular component of the GF vector. For the shower particles that predominantly contribute to the emitted Cherenkov light, the perpendicular component is comparable to the GF vector component perpendicular to the shower direction $\left(\mathbf{B}_{\perp}=\mathbf{B}-\left(\mathbf{B} \cdot \hat{z}^{\prime}\right) \hat{z}^{\prime}\right)$. Deflection of the shower particles by the GF breaks the azimuthal symmetry of the shower causing an elongation in the shower particle distribution in the plane orthogonal to $\mathbf{B}_{\perp}$.

Due to the asymmetry in the shower development induced by the GF, the Cherenkov light distribution observed by a telescope depends on both the distance to the shower impact position $(\rho)$ and the orientation of the shower impact vector relative to the GF. We parameterize the shower orientation with respect to telescope $i$ by the shower position angle $\left(\phi_{i}\right)$ defined by

$$
\cos \phi_{i}=\hat{x}^{\prime} \cdot \frac{\boldsymbol{\rho}_{i}}{\left|\boldsymbol{\rho}_{i}\right|} .
$$

Telescopes with a shower position angle of $0^{\circ}$ and $90^{\circ} \quad{ }_{648}$ view the shower in the planes parallel and perpendicular 649 to its elongated axis respectively (see Figure 5).

The shower reconstruction is performed in two con- 651 secutive stages. A geometric reconstruction algorithm 652 is first used to obtain a robust estimation of the shower ${ }_{653}$ parameters. In this stage the shower energy and inter- 654 action depth are initially assigned using look-up tables. 655 In the second stage the shower parameters derived from 656 the geometric reconstruction are refined using a likeli- 657 hood reconstruction algorithm that performs a joint fit 658 to the image intensity in all telescopes.

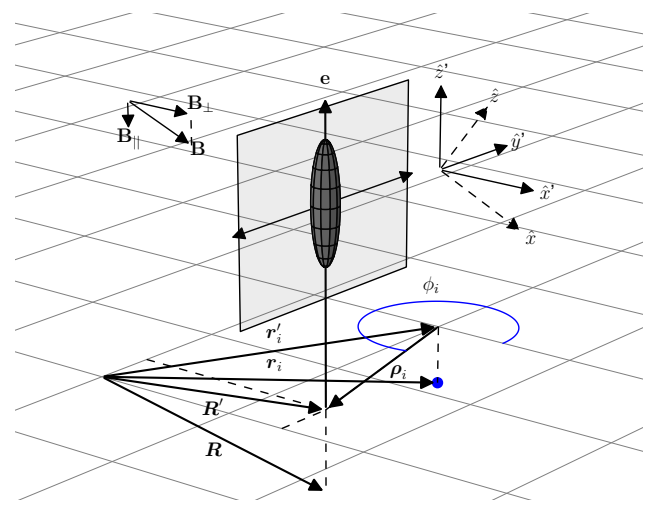

Figure 5: Illustration of the geometry of a gamma-ray shower as shown in the shower coordinate system. The gamma-ray trajectory is defined by its impact position $\mathbf{R}^{\prime}$ in the $x^{\prime}-y^{\prime}$ plane and arrival direction $\mathbf{e}$. The GF induces an elongation in the shower in the plane orthogonal to $\mathbf{B}_{\perp}$ (indicated by the grey shaded square). The shower impact vector, $\boldsymbol{\rho}_{i}$, describes the position of the shower impact position relative to the telescope at $\mathbf{r}_{i}$ (closed blue circle). The shower position angle, $\phi_{i}$, is defined by the angle between the shower impact vector and the $x^{\prime}$-axis.

\subsubsection{Geometric Reconstruction}

The geometric reconstruction algorithm is a 3-D stereoscopic reconstruction technique based on the traditional Hillas image parameterization of the shower images [35]. The emitted Cherenkov light from a gamma-ray shower produces an approximately elliptical distribution in the telescope focal plane with the major axis of the ellipse aligned with the shower trajectory. The projected shower trajectory as observed by a telescope with impact vector $\boldsymbol{\rho}_{i}$ can be described by the equation

$$
\mathbf{e}_{s, i}(t)=\frac{\rho_{i}+\mathbf{e} t}{\left|\rho_{i}+\mathbf{e} t\right|},
$$

where $t$ is the physical distance along the shower axis from its intersection with the shower plane. Each telescope that observes the shower constrains the trajectory to lie in the plane formed by the vectors $\hat{z}^{\prime}$ and $\rho_{i}$. When multiple telescope images are present, the intersection point of the projected shower axes provides a unique solution for both the shower direction (e) and its impact position in the shower plane $\left(\mathbf{R}^{\prime}\right)$.

The solution for the shower trajectory that best matches the projected shower axes of all telescopes is found by minimizing a pair of $\chi^{2}$-like parameters that independently optimize the shower direction and core position. In the case of the shower direction we solve 
for the vector e that minimizes

$$
\chi_{e}^{2}(\mathbf{e})=\sum_{i} \kappa\left(S_{i}, w_{i}, l_{i}\right) \Delta_{e, i}(\mathbf{e})^{2},
$$

where $\Delta_{e, i}(\mathbf{e})$ is the distance of closest approach between ${ }^{697}$ the major axis of the image ellipse and the shower direc- 698 tion projected to the image plane of telescope $i$, and $\kappa$ is ${ }^{699}$ a weighting function that controls the contribution of 700 each telescope to the total sum. Images that are brighter 701 and more elongated provide a better constraint on the 702 shower trajectory, and therefore we use as our weight- 703 ing function the product of the image size with square ${ }^{704}$ of the image ellipse eccentricity,

$$
\kappa\left(S_{i}, w_{i}, l_{i}\right)=S_{i} \frac{l_{i}^{2}-w_{i}^{2}}{l_{i}^{2}} .
$$

The shower core position is reconstructed by minimizing

$$
\chi_{R}^{2}(\mathbf{R})=\sum_{i} \kappa\left(S_{i}, w_{i}, l_{i}\right) \Delta_{R, i}(\mathbf{R})^{2},
$$

where

$$
\Delta_{R, i}(\mathbf{R})=\left|\boldsymbol{\rho}_{i}(\mathbf{R})-\left(\boldsymbol{\rho}_{i}(\mathbf{R}) \cdot \mathbf{e}_{\rho, i}\right) \mathbf{e}_{\rho, i}\right|
$$

is the distance of closest of approach between the image axis of telescope $i$ projected to the shower plane 715 $\left(\mathbf{e}_{\rho, i}\right)$ and the core location. After reconstruction of the 716 shower trajectory, the shower energy is reconstructed 717 using look-up tables for the shower energy as a function of the image size and impact distance from the telescope. The shower energy estimate is calculated from a weighted average of telescope energy estimates given by

$$
E=\left(\sum_{i} \sigma_{E}\left(S_{i}, \rho_{i}\right)\right)^{-1} \sum_{i} \frac{E\left(S_{i}, \rho_{i}\right)}{\sigma_{E}\left(S_{i}, \rho_{i}\right)},
$$

$$
\text { where } E\left(S_{i}, \rho_{i}\right) \text { and } \sigma_{E}\left(S_{i}, \rho_{i}\right) \text { are functions for the ex- }
$$
pectation value and standard deviation of the shower energy derived from simulations.

\subsubsection{Likelihood Reconstruction}

The likelihood reconstruction performs a global fit to the telescope image data using a model for the expected pixel amplitude $\mu(\boldsymbol{\theta})$ as a function of the shower parameters $\boldsymbol{\theta}$. Pixel expectation values are evaluated from an image template model, $I(\mathbf{e} ; \boldsymbol{\rho}, \boldsymbol{\theta})$, a probability distribution function for the image intensity in the direction $\mathbf{e} 724$ as measured by a telescope that observes a shower with 725 parameters $\boldsymbol{\theta}$ and impact vector $\boldsymbol{\rho}$. More details on the 726 generation of the image intensity model are presented in Section 3.2.3. The agreement between the telescope image model and the data is evaluated by means of an array likelihood function. Shower parameters are determined by a maximization of an array likelihood function. Maximization of the array likelihood as a function of shower fit parameters is performed using a numerical non-linear optimization technique. In order to ensure stable fit convergence, the shower parameters are initially seeded with a set of values derived by the geometric reconstruction $\left(\boldsymbol{\theta}_{\mathrm{geo}}\right)$.

We use a formulation of the array likelihood function which is similar to the one presented in [36]. The array likelihood is computed from a pixel-by-pixel comparison between the observed and predicted image intensities. The likelihood provides a statistical model for the measured pixel signal $(s)$ as a function of input models for signal and background. The measured pixel signal is modeled as the sum of three components: Cherenkov signal photons, NSB photons, and Gaussian noise arising from detector fluctuations. The pixel likelihood function is

$$
L_{\text {pix }}\left(s \mid \mu(\boldsymbol{\theta}), \mu_{b}, \sigma_{b}, \sigma_{\text {spe }}\right)=\sum_{n} \frac{\left(\mu+\mu_{b}\right)^{n} e^{-\left(\mu+\mu_{b}\right)}}{n !} g(s, n),
$$

where $\mu$ is the model amplitude, $\mu_{b}$ is the NSB amplitude, $\sigma_{b}$ is the standard deviation of the detector noise, $\sigma_{\text {spe }}$ is the width of the single PE response function, and

$$
g(s, n)=\frac{1}{\sqrt{2 \pi\left(\sigma_{b}^{2}+n \sigma_{\mathrm{spe}}^{2}\right)}} \exp \left[-\frac{(s-n)^{2}}{2\left(\sigma_{b}^{2}+n \sigma_{\mathrm{spe}}^{2}\right)}\right] .
$$

The model amplitude for pixel $j$ in telescope $i$ is calculated by an integration of the image template model over the pixel,

$$
\mu_{i j}(\boldsymbol{\theta})=\int_{\Omega_{i j}} I\left(\mathbf{e} ; \boldsymbol{\rho}_{i}, \boldsymbol{\theta}\right) d \Omega,
$$

where $\Omega_{i j}$ is the 2-D angular integration region.

The array likelihood is calculated from the product of the pixel likelihoods in all telescopes,

$$
L\left(\mathbf{s} \mid \boldsymbol{\mu}(\boldsymbol{\theta}), \mu_{b}, \sigma_{b}, \sigma_{\gamma}\right)=\prod_{i, j} L_{\mathrm{pix}}\left(s_{i j} \mid \mu_{i j}(\boldsymbol{\theta}), \mu_{b}, \sigma_{b}, \sigma_{\gamma}\right),
$$

where $s_{i, j}$ and $\mu_{i, j}$ are the signal and model amplitude of pixel $i$ in telescope $j$. The set of pixels included in the computation of Equation 19 can encompass the entire 
camera. Unlike for the geometric reconstruction techniques, each pixel is weighted by its expected contribution to the total image intensity. Therefore the inclusion of pixels on the shower periphery does not significantly improve or degrade the reconstruction performance. Although the array likelihood can be calculated using all pixels in the camera, using a smaller number of pixels significantly reduces the computation time needed for the shower likelihood optimization. In order to select pixels that will provide a useful constraint on the shower parameters, we choose a set of pixels $\mathcal{P}$ in each telescope that satisfies the relation

$$
\sum_{j \in \mathcal{P}} \mu_{j}\left(\boldsymbol{\theta}_{\text {geo }}\right) \geq f \sum_{j} \mu_{j}\left(\boldsymbol{\theta}_{\text {geo }}\right),
$$

where $\mu_{j}$ is the expected image intensity in pixel $j$ and $f$ is the fraction of the total image intensity. We build the set $\mathcal{P}$ by adding pixels in order of their expected intensity until the total amplitude fraction exceeds $f$. Having found that the reconstruction performance is relatively insensitive to $f$ for values $\gtrsim 0.75$, we use $f=0.75$.

An underlying assumption of the likelihood formulation presented here is that the shower can be treated as a continuous distribution of particles. However, because the electromagnetic cascade is a stochastic process, nonstatistical deviations from the image model are expected due to fluctuations in the shower development. These deviations become especially important at low energies where the total number of shower particles is small and the influence of the GF becomes large. These shower fluctuations will tend to worsen the performance of the method relative to what would be expected in the case of purely statistical fluctuations.

\subsubsection{Image Templates}

The image model, $I(\mathbf{e} ; \boldsymbol{\rho}, \boldsymbol{\theta})$, is the probability distri- 775 bution function for the measured telescope image inten- 776 sity in photoelectrons (PEs) versus direction, e. The 777 model is parameterized as a function of the shower 778 properties (energy and first interaction depth) and the 779 impact position of the shower relative to the telescope. 780 The model is generated by averaging the intensity of 781 a large sample of simulated showers generated at a se- 782 quence of fixed offsets, energies and interaction depths. 783 The image templates for this study were generated 784 with the CORSIKA shower simulation package and the 785 detector simulation described in Section 2.3. While 786 the image templates used for this study are MC-based 787 we note that the likelihood reconstruction can also be 788 applied using templates generated with semi-analytic 789 shower models [36].
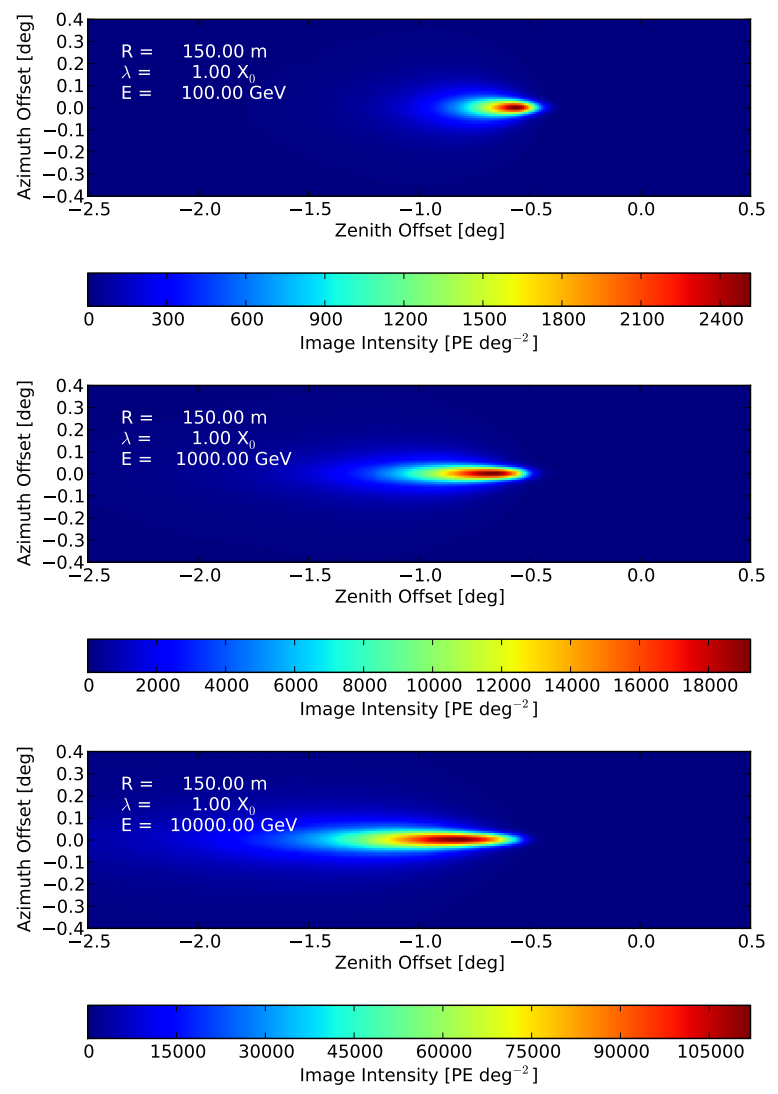

Figure 6: Image intensity templates for three different three gammaray energies $(100 \mathrm{GeV}, 1 \mathrm{TeV}$, and $10 \mathrm{TeV})$ generated for a telescope model with $R_{\mathrm{psf}}=0.02^{\circ}$ and $A_{\mathrm{opt}}=11.18 \mathrm{~m}^{2}$. The images show the expectation for the measured intensity of Cherenkov light as a function of angular offset from the primary gamma-ray direction. The image templates shown here are evaluated at an impact distance $(\rho)$ of $150 \mathrm{~m}$ and a first interaction depth $(\lambda)$ of $1 \mathrm{X}_{0}$.

Because the templates are produced from a simulation of the shower, the image model incorporates all effects that influence the measured image intensity including atmospheric attenuation, geomagnetic field, telescope optics, and telescope detector response. The image model is a continuous distribution for the shower photons in the focal plane and the same template can therefore be used to compute the image intensity for cameras with arbitrary pixel geometry and field-ofview. For this study, we use image templates computed for the baseline telescope model with $D=12 \mathrm{~m}$ and $A_{\text {opt }}=11.18 \mathrm{~m}^{2}$. The image intensity for other telescopes is calculated by rescaling the image intensity by the ratio of the telescope light collection area to the baseline telescope model.

The image intensity templates are stored on a sixdimensional grid: 
- $\log _{10}$ (Energy) and Interaction Depth $\left(\log _{10} E\right.$ and 841 ג)

- Core Impact Distance and Position Angle ( $\rho$ and $\phi$ )

- Projected Zenith and Azimuth Offset in Image Template Coordinates

The shower templates are defined in a coordinate system rotated by $\phi_{i}$ with respect to the shower axis such that 847 the $x$-axis is aligned with the shower axis. In order to 848 keep the memory footprint of the full six-dimensional 849 template at a manageable level, the image templates are 850 only defined over an angular region within one degree 851 of the shower axis.

The expected image intensity is computed from the ${ }_{853}$ image template sequence by a linear interpolation in the 854 six-dimensional template space. The templates are also 855 used to derive first derivatives of the image intensity as 856 a function of the shower parameters which are used for 857 calculation of the likelihood gradient.

Figure 6 shows the image templates evaluated for 859 gamma-ray showers of three different energies. The 860 primary energy affects both the total intensity of the 861 shower image as well as its shape. Higher energy show- 862 ers propagate further into the atmosphere and result in 863 shower images that are more extended along the shower 864 axis. The core impact distance sets the geometrical 865 perspective of the telescope and selects the Cherenkov light emission from particles with a specific range of angles with respect to the shower axis. Showers observed inside the Cherenkov light pool $(\rho \lesssim 120 \mathrm{~m})$ appear both brighter and narrower as the telescope ac- ${ }_{866}$ cepts Cherenkov light from higher energy particles that ${ }_{867}$ are closely aligned with the shower primary. More dis- ${ }_{868}$ tant showers are dimmer and increasingly offset from ${ }_{869}$ the primary origin. The interaction depth sets the start- ${ }_{870}$ ing point of the shower and primarily influences the displacement of the shower image along the shower axis.

In the absence of the GF, the shower template is symmetric with respect to the shower position angle. The GF breaks the axial symmetry as the Lorentz force preferentially perturbs the trajectory of the shower particles into the plane orthogonal to $\mathbf{B}_{\perp}$. The GF effect is especially pronounced for showers with small interaction depth for which the average propagation distance between the first and second interactions is large. Figure 7 illustrates the impact of the GF on the image template for three values of the core position angle $(\phi)$ : $0^{\circ}$ (parallel), $45^{\circ}$, and $90^{\circ}$ (perpendicular). The shower width monotonically decreases as the shower position angle is increased from $0^{\circ}$ to $90^{\circ}$ reflecting the asymmetry in the shower development. For intermediate viewing angles $\left(\phi=45^{\circ}\right)$, the GF also causes a rotation of the image major axis relative to the shower axis

\subsection{Gamma/Hadron Separation and Cut Optimization}

The final stage of the event analysis determines parameters that can be used for discrimination between cosmic- and gamma-ray initiated air showers. The Cherenkov images produced by cosmic-ray showers can generally be distinguished from gamma-ray showers by their wider and more irregular appearance. Hadronic subshowers may also produce isolated clusters of Cherenkov light in the telescope image plane. A widely used set of parameters for background discrimination are the so-called mean scaled parameters defined already in the HEGRA collaboration [34] and extensively used by current generation IACT experiments (see e.g., [37]). The mean scaled parameters provide a measure the deviation between the observed and expected telescope image moments for a gammaray shower. Using a set of simulated gamma-ray showers, lookup tables for the mean and standard deviation of the image moment parameters are produced as a function of the telescope image size and telescope impact distance (denoted here as $p(S, \rho)$ and $\sigma_{p}(S, \rho)$ ). For a telescope image parameter $p_{i}$ we define the array-level parameter as

$$
p=\left(\sum_{i} w_{i}\right)^{-1} \sum_{i} w_{i} \frac{p_{i}-p\left(S_{i}, \rho_{i}\right)}{\sigma_{p}\left(S_{i}, \rho_{i}\right)},
$$

where the sum is over all telescopes with reconstructed image parameters and $w_{i}$ is a weighting factor. We use $w_{i}=S_{i} / \sigma_{p}\left(S_{i}, \rho_{i}\right)$ assigning a larger weight to telescopes with brighter images and a smaller expected dispersion in the image parameter.

A second class of discriminant variables can be obtained by computing a goodness-of-fit between the data and the image template model evaluated at the best-fit shower parameters [36]. When considering Gaussiandistributed data the natural goodness-of-fit parameter is the $\chi^{2}$ statistic. For the purposes of background discrimination, it is not critical to have an exact model for the asymptotic distribution of the test statistic as long as it provides good separation power between signal and background. To quantify the agreement between the measured and expected pixel signals we define a $\chi^{2}$-like parameter which we call the goodness-of-fit,

$$
\mathcal{G}=\frac{1}{N} \sum_{i} \sum_{j \in \mathcal{P}_{i}} \frac{\left(s_{i, j}-\mu_{i, j}(\boldsymbol{\theta})\right)^{2}}{\mu_{i, j}(\boldsymbol{\theta})+\mu_{b}},
$$



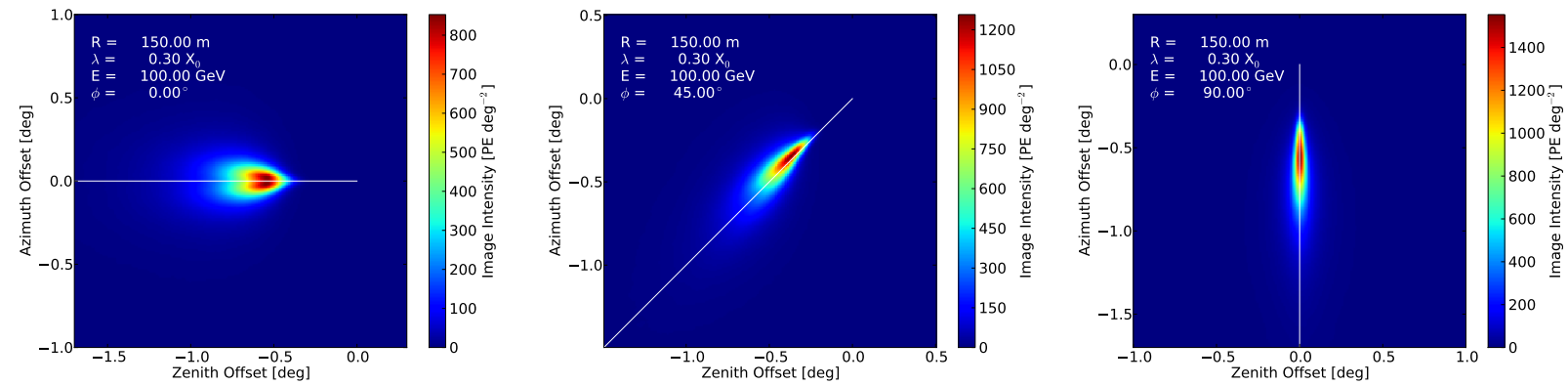

Figure 7: Image intensity templates as a function of angular offset from the primary gamma-ray direction for three values of the shower position angle: $\phi=0^{\circ}, \phi=45^{\circ}, \phi=90^{\circ}$. The templates shown are evaluated for a gamma-ray shower with an energy of $100 \mathrm{GeV}$, an impact distance of $150 \mathrm{~m}$, and an interaction depth of $0.3 \mathrm{X}_{0}$. The solid white line in each image shows the projection of the primary trajectory to the image plane.

where $\mathcal{P}_{i}$ is a set of pixels in telescope $i$ and $N$ is the ${ }_{918}$ total number of pixels in the summation. We found that the best separating power was achieved by evaluating Equation 22 using the set of telescope pixels that survive image cleaning, which we refer to as the image goodness-of-fit.

To maximally exploit the rejection power drawn from the ensemble of event parameters we further make use of boosted decision trees (BDTs) generated with the TMVA package [38]. The use of machine learning techniques have been shown to provide significant improvement in overall background rejection power when applied to IACT data [39]. We specifically use BDTs trained with the GradBoost algorithm with 200 trees, a maximum depth of 8 , and a shrinkage parameter of 0.1 . We train the decision tree analysis using the following six parameters: mean scaled width, mean scaled length, mean scaled displacement, array core distance, first interaction depth, and image goodness-of-fit. In order to avoid overtraining we use a training data set that constitutes $20 \%$ of the total gamma-ray and proton Monte Carlo samples.

\section{Results}

Using the simulation and analysis frameworks de- 941 scribed in Sections 2 and 3, we have explored the in- 942 fluence of the telescope design on the sensitivity and 943 gamma-ray reconstruction performance of various array 944 design concepts. Section 4.1 outlines the performance 945 metrics used for comparison of the arrays. Section 4.2996 defines a reference array alongside several benchmark 947 configurations which are representative of realistic tele- 948 scope and array configurations that will be chosen for 949 CTA. In the subsequent sections we examine the influ- 950 ence of each telescope parameter on the array perfor- 951 mance. In Section 4.12 we study the performance of the 952 benchmark arrays

\subsection{Performance Metrics and Cut Optimization}

Our primary metric for the comparison of different array and telescope designs is the differential gamma-ray point-source sensitivity evaluated following the standard procedure for CTA-related studies [18]. The differential sensitivity is evaluated in a sequence of logarithmic bins of reconstructed energy with a width of 0.2 dex (five bins per decade of energy). In each energy bin we calculate the expected number of signal and background events within an energy-dependent aperture of radius $\theta$. The number of signal events is estimated assuming a gamma-ray point-source in the center of the FoV. The residual background rate is estimated by scaling the number of background events reconstructed in the inner $3^{\circ}$ of the camera to the gamma-ray extraction area. In each bin we require a $5 \sigma$ excess above background and at least 10 signal events. The source significance is calculated using the method of [40] and a signal-free background region with a solid angle five times larger than the signal aperture. We further require a signal with a fractional amplitude above background of $5 \%$ in order to account for systematic errors in background estimation.

Sensitivity to spatially extended gamma-ray sources is calculated following the same procedure but with the gamma-ray signal spread out uniformly over a disk with angular diameter $D$. For a source with a given flux, the diffuse source sensitivity is always worse than the pointsource sensitivity. In the case of a point-source, the sensitivity depends on both background rejection efficiency and the PSF. The diffuse-source sensitivity, however, depends primarily on the background rejection efficiency and is nearly independent of the PSF when $D$ is larger than the PSF. 
The quality of the gamma-ray reconstruction is estimated from the simulated gamma-ray PSF, shower core resolution, and energy resolution. The most important of these quantities is the gamma-ray PSF as it directly impacts the sensitivity to point-sources and the morphology of extended gamma-ray sources. We characterize the gamma-ray PSF by the radius that contains $68 \%$ of the distribution of reconstruction errors $(68 \%$ containment radius).

When evaluating the performance of an array we apply several selection criteria to reject both background events and gamma-ray events with poor reconstruction quality. Point-source cuts are composed of two energydependent selections on the gamma/hadron rejection parameter and aperture radius, $\xi(E)$ and $\theta(E)$, parameterized as a cubic spline. The shape of these parameterizations is independently optimized for each array 1005 and exposure time to maximize the differential point- 1006 source sensitivity versus energy. At high energies where ${ }_{1007}$ the sensitivity of IACT arrays transitions from being 1008 background- to signal-limited, the optimal point-source ${ }_{1009}$ sensitivity is obtained by increasing the gamma-ray effi- ${ }_{1010}$ ciency and including events with poorer reconstruction ${ }_{1011}$ quality and a higher background contamination level. ${ }_{1012}$ Diffuse-source cuts are used when evaluating diffuse ${ }_{1013}$ source sensitivity and comprise the same selection on ${ }_{1014}$ the gamma/hadron parameter but with the aperture size ${ }_{1015}$ fixed to the radius of the source $(\theta(E)=D / 2)$.

Reconstruction cuts are used to define a homoge- ${ }_{1017}$ neous sample of well-reconstructed showers with core locations within a predefined fiducial area of the array. Showers passing reconstruction cuts must have an im- 1018 pact distance from the array center that is less than 1.2 times the distance from the center of the array to the 1019 nearest point along the array edge (as defined by the 1020 outer ring of telescopes). Showers with core locations 1021 near or within the array boundary (contained events) 1022 are sampled by a large number of telescopes that view 1023 the shower from multiple perspectives and allow for a 1024 more precise stereoscopic reconstruction of the shower 1025 trajectory. In arrays with mean telescope separations 1026 on par with the Cherenkov light pool size, contained ${ }_{1027}$ events will also have one or more telescopes that sample 1028 the shower within its Cherenkov light pool, where the 1029 Cherenkov light from the highest energy shower parti- 1030 cles is visible. The light emitted from these particles 1031 provides a much better constraint on the shower trajec- 1032 tory than the light emitted by lower energy shower par- 1033 ticles. Events outside the array boundary (uncontained ${ }_{1034}$ events) are sampled by a smaller number of telescopes 1035 for which the viewing angles are more closely aligned. ${ }_{1036}$ This results in a less precise determination of the shower ${ }_{1037}$
Table 1: Geometrical characteristics and optical performance of the camera and optical systems of the DC-MST, SC-MST, and LST telescope designs chosen for the prod-2 MC design study [33]. The offaxis PSF performance is evaluated at a field angle equal to $3 / 4$ of the distance to the edge of the FoV. For the LST and DC-MST designs which have cameras composed of hexagonal pixels, the given value of $D_{\text {pix }}$ is the width of a square pixel with the same solid angle as the hexagonal pixel used in that design.

\begin{tabular}{l|ccc} 
& LST & SC-MST & DC-MST \\
\hline$D_{\text {pix }}\left[{ }^{\circ}\right]$ & 0.084 & 0.067 & 0.171 \\
$A_{M}\left[\mathrm{~m}^{2}\right]$ & 412 & 50 & 100 \\
$A_{\text {opt }}\left[\mathrm{m}^{2}\right]$ & 52.5 & 7.29 & 13.65 \\
$R_{\text {psf }}\left[{ }^{\circ}\right]$ (on-axis) & 0.03 & 0.04 & 0.04 \\
$R_{\text {psf }}\left[{ }^{\circ}\right]$ (off-axis) & 0.12 & 0.04 & 0.08 \\
FoV $\left[{ }^{\circ}\right]$ & 4.5 & 8 & 8
\end{tabular}

trajectory.

Reconstruction cuts provide a measure of the gammaray reconstruction performance that can be evaluated independently of the source strength and exposure time. Relative to point-source cuts, reconstruction cuts offer worse point-source sensitivity but a significantly better gamma-ray PSF at high energies (above $1 \mathrm{TeV}$ ). The improvement in the gamma-ray PSF can be attributed to the removal of uncontained events which are bright enough to trigger the array at high energies. This selection is very useful when studying strong sources to check morphology and spectral features while not relying on the best signal-to-noise ratio.

\subsection{Benchmark Arrays}

The baseline CTA concept is an array of 50-100 telescopes distributed over an area of $\sim 4-5 \mathrm{~km}^{2}$ and composed of small-, medium-, and large-sized telescopes. Previous simulation studies have found that intermediate layouts with a few (3-4) large-sized, on the order of 20 medium-sized, and 50-60 small-sized telescopes offers the best tradeoff in performance over the full energy range of CTA [18]. Table 1 shows the primary characteristics of the currently considered designs for the large- and medium-sized telescopes. The LST design is optimized for sensitivity at gamma-ray energies below $100 \mathrm{GeV}$ and features a large effective mirror area which enables efficient triggering and reconstruction of low energy showers. The DC-MST and SC-MST are two alternative designs for the medium-sized telescope that would provide sensitivity in the core energy range of CTA $(100 \mathrm{GeV}-10 \mathrm{TeV})$. The DC-MST is a single dish telescope that is similar in overall design to current generation IACTs with a camera composed of hexago- 


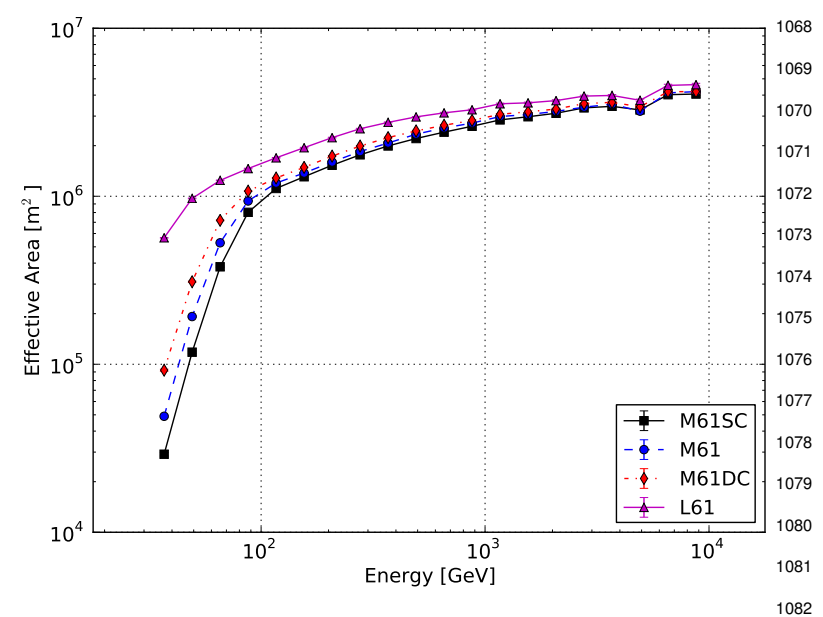

Figure 8: Trigger effective area versus gamma-ray energy for arrays ${ }^{1}$ M61SC, M61, M61DC, and L61. The camera trigger thresholds $\left(T_{\mathrm{th}}\right){ }^{1084}$ for these arrays are 45, 60, 80, and $123 \mathrm{PE}$ respectively.

nal pixels with flat-to-flat spacing of $0.184^{\circ}$. ${ }^{3}$ The SCMST employs the dual-mirror Schwarzschild-Couder optical design and uses a smaller pixel size of $0.067^{\circ}$ to achieve higher resolution imaging of the gamma-ray shower. Ray-tracing simulations of the SC-MST OS with realistic alignment tolerances for the mirrors and camera focal plane have demonstrated an optical PSF with a $68 \%$ containment radius between $0.02^{\circ}$ and $0.04^{\circ}$ [21]. Although the array designs considered for previous MC studies only incorporated DC-MSTs, the higher angular resolution SC-MST provides a potentially compelling option for the medium-sized CTA telescope.

We consider several different benchmark array configurations shown in Table 2 to explore the performance of different array and telescope designs for CTA. M61 is a reference array configuration with an effective light collection area that is intermediate between the DC- and SC-MST designs and an imaging performance similar to the SC-MST. M61SC is an array configuration with the same imaging performance as M61 but with a reduced light collection area that is more comparable to the SC-MST design. M61DC and M25DC are chosen to represent a 61 and 25 telescope array respectively composed of telescopes of the DC-MST design. The latter configuration corresponds to the number of MSTs in the baseline CTA design [18]. Arrays L5 and L61 are composed of telescopes with an optical effective area comparable to the LST design and an imaging performance similar to the SC-MST.

We show in Figure 8 the trigger effective area for ar-

\footnotetext{
${ }^{3}$ Equivalent in solid angle to square pixels with $D_{\text {pix }}=0.171^{\circ}$.
}

rays M61, M61SC, M61DC, and L61. The camera trigger threshold of each array is set using the telescope effective light collection area and Equation 4 The sharp downturn in the effective area of the MST arrays around $100 \mathrm{GeV}$ can be attributed to the onset of the trigger energy threshold. Below the trigger threshold energy, the image amplitude of an average gamma-ray shower is insufficient to trigger telescopes within the Cherenkov light pool. At these energies only showers with large interaction depth can be effectively recorded, and the total effective area is primarily determined by the trigger efficiency for contained showers that impact within the array perimeter. At higher energies all of the arrays become fully efficient for triggering contained showers and differences in the effective area arise predominantly from the efficiency for detecting showers around the array perimeter. As the shower energy increases, the area over which the arrays are fully efficient continues to increase and eventually extends well beyond the physical footprint of the array. Relative differences in the effective area for telescopes with different $A_{\text {opt }}$ are significantly smaller at the highest energies as gains in the effective area only come from showers around the array perimeter.

\subsection{Comparison with Other Telescope Simulations}

The simplifications in the detector response of the FAST simulation yield a much faster simulation code and enables the study of a broader parameter space compared to more detailed telescope simulations. Our simplified telescope model also enables us to employ a shower likelihood model which is nearly perfectly matched to the response characteristics of the telescopes. These simplifications allow us to explore the theoretical limit of the performance achievable by an IACT array when all characteristics of the telescope optics and camera are accounted for in the event reconstruction.

We have assessed the impact of the simplifications made in the FAST tool on the derived point-source sensitivity and gamma-ray PSF by comparing FAST against the well-tested sim_telarray package. We use both packages to simulate a 61 telescope array with the same geometry as our benchmark array layout with $120 \mathrm{~m}$ inter-telescope separation. For the sim_telarray simulation we use the prod-2 SCT model [33] with a trigger pixel threshold of 3.1 PE. For the FAST simulations, we use a telescope model with the same performance characteristics as the prod-2 SCT model shown in Table 1 and a camera trigger threshold of 42 PE. Relative to the telescope model used for the M61SC benchmark array, the prod-2 SCT model has a 
Table 2: Number of telescopes and telescope model parameters of the benchmark array configurations used for this study. All arrays are composed of telescopes arranged on a uniform grid with constant inter-telescope spacing of $120 \mathrm{~m}$ as shown in Figure 1 .

\begin{tabular}{l|cccccc} 
Name & $\mathrm{N}_{\text {tel }}$ & $A_{\text {opt }}\left[\mathrm{m}^{2}\right]$ & $D_{\text {pix }}\left[^{\circ}\right]$ & $\left.R_{\text {psf }}{ }^{\circ}\right]$ & $T_{\text {th }}[\mathrm{PE}]$ & $R_{\text {nsb }}[\mathrm{MHz}]$ \\
\hline M61 & 61 & 11.18 & 0.06 & 0.02 & 60 & 14.7 \\
M61SC & 61 & 8.38 & 0.06 & 0.02 & 45 & 11.1 \\
M61DC & 61 & 14.91 & 0.16 & 0.08 & 80 & 139.5 \\
M25DC & 25 & 14.91 & 0.16 & 0.08 & 80 & 139.5 \\
L5 & 5 & 47.15 & 0.06 & 0.02 & 123 & 61.9 \\
L61 & 61 & 47.15 & 0.06 & 0.02 & 123 & 61.9
\end{tabular}
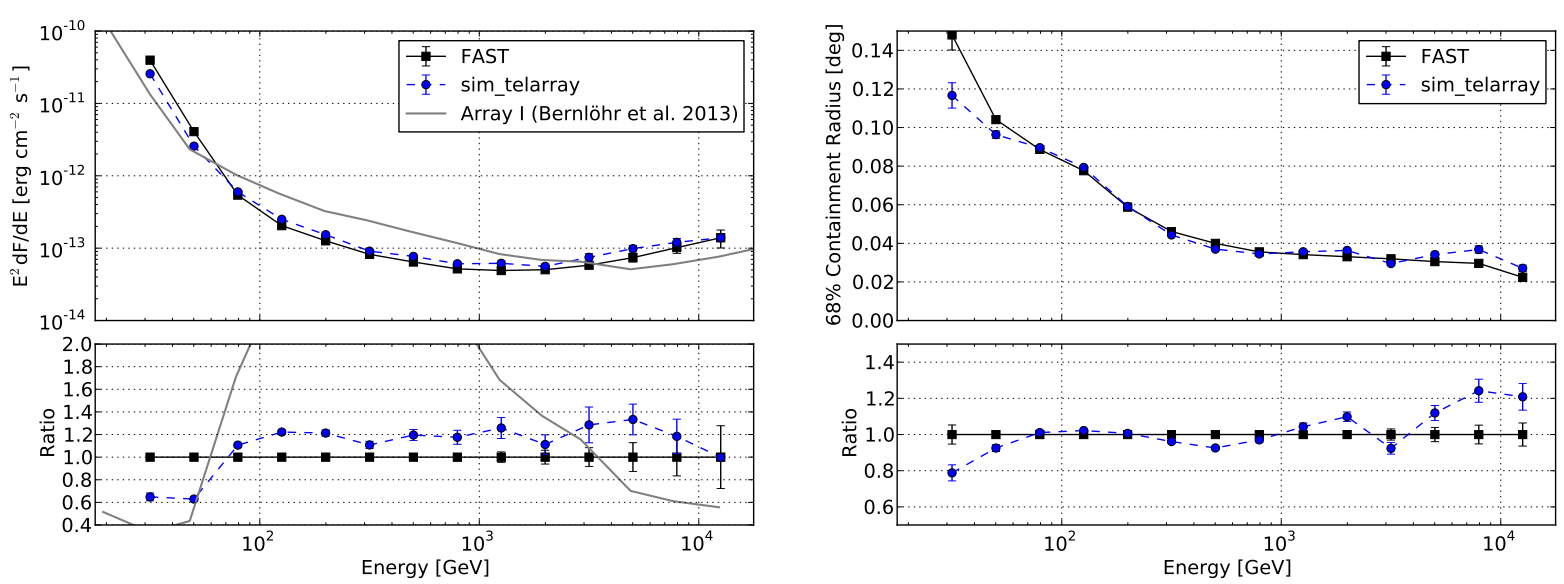

Figure 9: Performance of a 61 telescope array simulated with FAST (black squares) and sim_telarray (blue circles). Left: Differential pointsource sensitivity for a $50 \mathrm{~h}$ observation time. Shown as the dashed black line is the differential sensitivity of Array I from [18] evaluated with the most sensitive analysis at each energy. Right: $68 \%$ containment radius of the gamma-ray PSF after point-source cuts.

larger pixel size and optical point-spread function and ${ }_{1141}$ a slightly smaller light collection area. For gamma-ray ${ }_{1142}$ showers near the trigger threshold $(\mathrm{E} \sim 100 \mathrm{GeV})$, the ${ }_{1143}$ sim_telarray telescope model has a slightly lower ef- 1144 fective camera threshold as compared to the telescope 1145 in our simplified simulations. The choice of a higher ${ }_{1146}$ threshold for our simulations was made to be conser- 1147 vative and limit possible overestimations in sensitivity ${ }_{1148}$ close to the threshold.

Fig. 9 shows the comparison of the array perfor- ${ }^{1150}$ mance obtained when simulating the same array with ${ }^{1151}$ sim_telarray and FAST. We include in the same fig- ${ }^{1152}$ ure the point-source sensitivity of Array I from [18] ${ }^{1153}$ which was simulated using sim_telarray but with a ${ }^{1154}$ different array and telescope setup. At energies above $75 \mathrm{GeV}$ the point-source sensitivity obtained with the ${ }^{1155}$ FAST simulations is $20 \%$ better than the sim_telarray 1156 simulations. The gamma-ray PSF and gamma-ray re- 1157 construction efficiency is similar over the same energy 1158 range indicating that the improvement in point-source 1159 sensitivity can be attributed to an enhanced gamma- 1160 hadron separation in the FAST simulations. Below 1161
$50 \mathrm{GeV}$ the sim_telarray simulations yield a $40 \%$ better sensitivity due to the slightly lower telescope trigger threshold. Although we observe measurable differences in the array performance when comparing our simulations with sim_telarray, the differences in point-source sensitivity are much smaller than the differences between individual analysis packages that use the same sim_telarray simulations as input (see e.g. the comparison of alternative analyses in [18]). Furthermore the conclusions drawn in this work about the relative performance of different array and telescope designs is most likely not affected by these differences. It is thus easy to scale our results and readily compare them to other sim_telarray results.

\subsection{Performance of the Likelihood Reconstruction}

Relative to moment-based reconstruction techniques, likelihood-based reconstruction algorithms have been shown to provide better gamma-ray angular resolution as well as improved separation between gamma-ray and cosmic-ray induced showers [41, 36, 18]. We assess the relative improvement from the likelihood ap- 

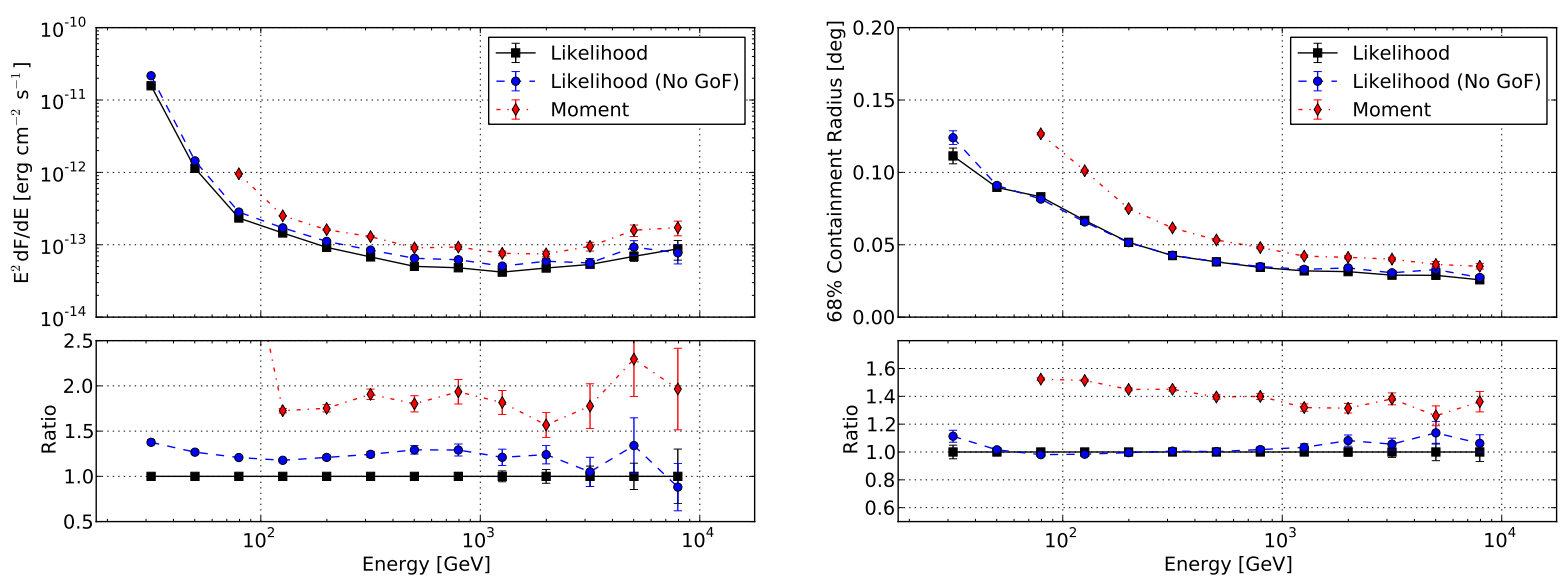

Figure 10: Reconstruction performance and gamma-ray point-source sensitivity of array M61 obtained with different event reconstruction and analysis algorithms: likelihood (black, solid), likelihood without goodness-of-fit (blue, dashed), and moment (red, dot-dashed). Left: Differential point-source sensitivity for a $50 \mathrm{~h}$ observation time. Right: $68 \%$ containment radius of the gamma-ray PSF after point-source cuts.

proach by comparing its performance with an analysis 1194 that uses only the geometric trajectory reconstruction 1195 and moment-based image parameterization described in 1196 Section 3.2 which we refer to here as the moment re- 1197 construction. Because the moment reconstruction is 1198 more sensitive to the presence of noise fluctuations in 1199 the image, we use a slightly higher cleaning thresh- 1200 old $(\bar{s} / \sigma=9)$ than the threshold used for the likelihood analysis. We use a BDT background discriminant ${ }_{1201}$ trained with the same settings described in Section 3.3 but excluding parameters derived from the likelihood ${ }^{1202}$ analysis.

Figure 10 shows the comparison of the point-source sensitivity obtained with the moment analysis, the likelihood analysis, and a likelihood analysis in which the goodness-of-fit (GOF) parameter is excluded from the training of the decision tree. With the likelihood-based analysis, we find a factor of two improvement in pointsource sensitivity and a $30-40 \%$ improvement in the gamma-ray PSF over the full energy range. As seen from the comparison between the likelihood analyses performed with and without the GOF parameter, the improvement in the point-source sensitivity is attributable to gains in both the gamma-ray angular resolution and the background rejection power. The addition of the ${ }_{1217}$ GOF parameter provides an additional 30\% improve- ${ }_{1218}^{1217}$ ment in sensitivity.

We also observe that the energy threshold for the like- 1220 lihood analysis is considerably lower $\left(E_{\mathrm{th}} \simeq 50 \mathrm{GeV}\right){ }_{1221}$ relative to the moment reconstruction $\left(E_{\mathrm{th}} \simeq 100 \mathrm{GeV}\right)$. 1222 The improved performance of the likelihood analysis at ${ }_{1223}$ low energies can be attributed to both the higher im- 1224 age reconstruction efficiency and the smaller bias of the likelihood energy estimator. Because the likelihood reconstruction is insensitive to the inclusion of pixels with small signals, the cleaning threshold can be optimized to maximize the reconstruction efficiency for low-energy showers without impacting the performance at higher energies.

\subsection{Influence of the Geomagnetic Field}

The deflection of charged particles in the EM shower by the geomagnetic field (GF) can significantly distort the shapes of gamma-ray images recorded by IACTs [42, 43, 44]. The strength and orientation of the GF is thus an important consideration for the selection of candidate sites for an IACT observatory. Its influence can be as large or larger than the site elevation [45]. The magnitude of the induced deflection is proportional to the perpendicular component of the GF $\left(B_{\perp}\right)$ and therefore the strength of the GF effect depends on both the magnitude of the GF vector as well as its orientation relative to the shower trajectory. Due to the asymmetry in the shower shape induced by the GF, the distortion visible to a telescope also depends on the orientation of the shower impact point relative to the telescope position. Telescopes with shower position angles close to $0^{\circ}$ or $180^{\circ}$ see a larger GF effect as the GF-induced elongation in the shower occurs primarily in the plane perpendicular to the telescope pointing.

To obtain a realistic assessment of the GF effect for any given observatory site would require simulations with many telescope orientations as they occur for realistic observation profiles of gamma-ray sources. We 

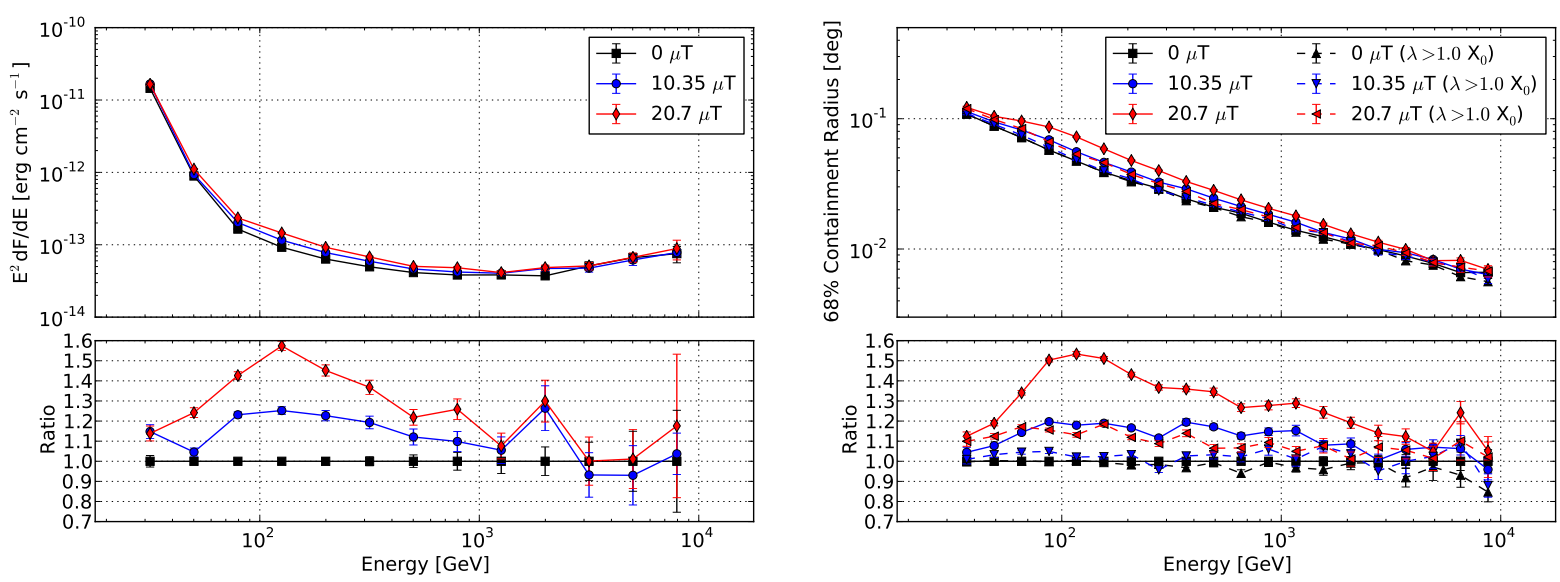

Figure 11: Performance of array M61 simulated with the equatorial GF $\left(B_{\perp}=20.7 \mu \mathrm{T}\right.$; red diamonds and solid line $)$, a GF configuration with a reduced perpendicular component $\left(B_{\perp}=10.35 \mu \mathrm{T}\right.$; blue circles and solid line), and no GF (black squares and solid line). Left: Differential point-source sensitivity for a $50 \mathrm{~h}$ observation time. Right: Gamma-ray angular resolution (68\% containment radius) after reconstruction cuts. Dashed curves show the same comparison for gamma-ray showers with an interaction depth $(\lambda)$ greater than $1.0 \mathrm{X}_{0}$.

did not carry out such simulations and instead focused ${ }_{1256}$ on the effect of the GF for a few representative values ${ }_{1257}$ of $B_{\perp}$. Our baseline site configuration has $\left(B_{x}, B_{z}\right)={ }_{1258}$ (27.5 $\mu \mathrm{T},-15.0 \mu \mathrm{T})$ with $B_{\perp}=20.7 \mu \mathrm{T}$ when observ- ${ }_{1259}$ ing a shower with $Z n=20^{\circ}$ and $A z=0^{\circ}$. To test the ${ }_{1260}$ influence of the GF strength we performed simulations ${ }_{1261}$ of array M61 for two additional site models: a site with ${ }_{1262}$ $\left(B_{x}, B_{z}\right)=(19.84 \mu \mathrm{T},-24.24 \mu \mathrm{T})$ that has a perpendicu- ${ }_{1263}$ lar GF component that is half as large as for our baseline ${ }_{1264}$ site $\left(B_{\perp}=10.35 \mu \mathrm{T}\right)$ and a site with no geomagnetic ${ }_{1265}$ field. By limiting ourselves to these few cases we can ${ }_{1266}$ only give a general guidance for effects of the magnetic ${ }_{1267}$ field on any observable gamma-ray source. Depending ${ }_{1268}$ on the specific source observation profile, the effect of 1269 the GF for an individual source might be different. $\quad{ }_{1270}$

The configurations we tested have a range of ${ }^{1271}$ field strengths that are comparable to the southern ${ }_{1273}$ Hemisphere sites considered for CTA. The Namib- ${ }_{1274}$ ian H.E.S.S. site and the Argentinian Leoncito sites ${ }_{1275}$ have $\left(B_{x}, B_{z}\right)=(12.1 \mu \mathrm{T},-25.5 \mu \mathrm{T})$ and $\left(B_{x}, B_{z}\right)={ }_{1276}$ $(20.1 \mu \mathrm{T},-12.2 \mu \mathrm{T})$, respectively [45]. Because the ${ }_{1277}$ strength and orientation of the GF is generally a slowly ${ }_{1278}$ varying function of the site latitude and longitude these ${ }_{1279}$ two sites provide a good representation of the expected ${ }_{1280}$ GF effect for sites in Africa and South America. When ${ }_{1281}$ observing a shower at $Z n=20^{\circ}$ and $A z=0^{\circ}$ the Namibian and Argentinian sites have perpendicular components of $2.7 \mu \mathrm{T}$ and $14.7 \mu \mathrm{T}$. However a more realistic measure of the expected GF effect is the average perpen- ${ }^{2} 283$ dicular component over the range of azimuth angles that ${ }_{1284}$ a gamma-ray source is observed. The Namibian and Ar- 1285 gentinian sites have an average GF strength at $Z n=20^{\circ}$ of $13.4 \mu \mathrm{T}$ and $19.7 \mu \mathrm{T}$, respectively.

The comparison of the array performance for the three GF configurations is presented in Figure 11. We find that the effect of the GF strength is strongest at $100 \mathrm{GeV}$ where the point-source sensitivity is reduced by $50 \%$ when increasing $B_{\perp}$ from $0 \mu \mathrm{T}$ to $20.7 \mu \mathrm{T}$. We also observe that the effect of the GF scales linearly with $B_{\perp}$ such that the site configuration with $B_{\perp}=10.35 \mu \mathrm{T}$ suffers approximately half of the reduction in sensitivity relative to our baseline site configuration. Below energies of $100 \mathrm{GeV}$, the effect of the GF is lessened because only gamma rays that convert deep in the atmosphere can be efficiently reconstructed. The lower the particle interacts in the atmosphere the less it is affected by the GF. At higher gamma-ray energies the impact of the GF is lessened due to both the higher energy of the secondary particles and the larger path length in the atmosphere. As shown in the right panel of Figure 11 the GF worsens the point-source sensitivity primarily by degrading the gamma-ray PSF. For showers with interaction depths larger than $1 \mathrm{X}_{0}$, differences in the gamma-ray PSF between the different GF configurations are found to be less than $20 \%$ illustrating that the influence of the GF increases with decreasing interaction depth.

\subsection{Imaging Performance}

The telescope design has a large impact on the resulting gamma-ray PSF obtained with the complete array. The optical design of the individual telescopes defines 

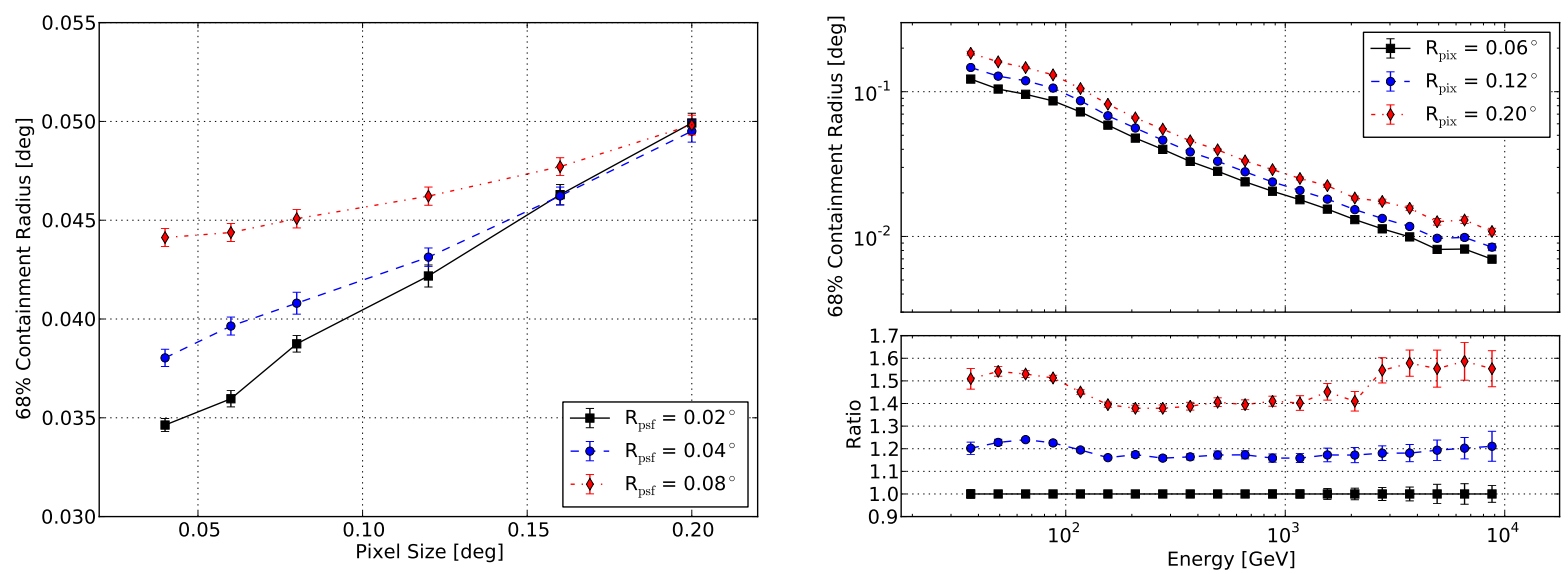

Figure 12: Left: $68 \%$ containment angle of the gamma-ray PSF at $317 \mathrm{GeV}$ versus camera pixel size for telescope models with different optical PSFs $\left(R_{\mathrm{psf}}\right): 0.02^{\circ}$ (black squares), $0.04^{\circ}$ (blue circles), $0.08^{\circ}$ (red diamonds). The gamma-ray PSF is evaluated after applying reconstruction cuts. The baseline configuration for all simulations is array M61. Right: 68\% containment angle of the gamma-ray PSF versus gamma-ray energy for array M61 with $R_{\mathrm{psf}}=0.02^{\circ}$ simulated with different telescope pixel sizes: $0.06^{\circ}$ (black squares), $0.12^{\circ}$ (blue circles), $0.20^{\circ}$ (red diamonds).

their achievable optical PSF and the camera design de- 1317 termines how efficiently the optical PSF can be trans- 1318 lated into an improved gamma-ray PSF. For a given op- 1319 tical PSF, the gamma-ray PSF can be improved by re- 1320 ducing the camera pixel size. In the limit that the pixel 1321 size is much smaller than the PSF, the improvement of ${ }_{1322}$ the gamma-ray PSF saturates and a further reduction in ${ }_{1323}$ pixel size does not provide any measurable advantage ${ }_{1324}$ but increases the cost of the camera. Thus the optimal ${ }_{1325}$ tradeoff between performance and cost is one in which 1326 the pixel size is appropriately matched to the quality of ${ }_{1327}$ the optical PSF. Current generation IACTs have cameras 1328 using pixel sizes from $0.1^{\circ}$ to $0.16^{\circ}$ and an optical PSF ${ }_{1329}$ at the center of the FoV which is considerably smaller ${ }_{1330}$ than the pixel size. Here we explore a new parameter ${ }_{1331}$ space for the IACT imaging resolution by examining the ${ }_{1332}$ performance of camera designs with pixel sizes between ${ }_{133}$ $0.04^{\circ}$ and $0.1^{\circ}$. Such designs begin to resolve the core ${ }_{1334}$ of the Cherenkov shower which has an intrinsic angular ${ }_{1335}$ size of $\sim 0.01^{\circ}$.

The left panel of Fig 12 shows the gamma-ray PSF ${ }^{1337}$ versus pixel size for arrays with different optical PSFs. For an optical PSF of $0.08^{\circ}$ the gamma-ray PSF shows only a modest improvement of $\sim 10 \%$ when reducing ${ }_{1341}$ the pixel size from $0.2^{\circ}$ to $0.04^{\circ}$. An optical PSF be- ${ }_{1342}$ tween $0.02^{\circ}$ and $0.04^{\circ}$ is found to be critical to real- ${ }_{1343}$ ize the full improvement in gamma-ray PSF that can be ${ }_{1344}$ achieved by reducing the camera pixel size below $0.12^{\circ}$. The improvement of the gamma-ray PSF at different en- 1345 ergies when reducing the pixel size is shown in Fig. 12 1346 The gamma-ray PSF is significantly better at all ener- ${ }_{1347}$ gies when reducing the pixel size. There is a slight modulation seen in the improvement versus energy more pronounced for larger pixel sizes. The smaller pixel size performs best at low and high energies $(E<100 \mathrm{GeV}$ and $E>2.5 \mathrm{TeV}$ ) while the improvement is less pronounced in the intermediate energy range. An improvement of the gamma-ray PSF of about 20\% in the full energy range by reducing the pixel diameter from $0.12^{\circ}$ to $0.06^{\circ}$ demonstrates a realistic difference between currently considered optical telescope designs for CTA.

The effect of the pixel size on the differential pointsource sensitivity is shown in Fig 13 The pixel size has the strongest impact at low energies $(<100 \mathrm{GeV})$ where a factor of two relative improvement is observed when the pixel size is reduced from $0.16^{\circ}$ to $0.06^{\circ}$. At higher energies the smaller pixel size results in a smaller but still measurable improvement in point-source sensitivity of $30-40 \%$. Above $3 \mathrm{TeV}$ differences between adjacent pixel sizes become indistinguishable due to the limited background statistics that make evaluation of small sensitivity differences very difficult. The gammaray PSF is clearly improved over the complete energy range by about $50 \%$ as the pixel size is reduced from $0.2^{\circ}$ to $0.06^{\circ}$. The observed improvement in sensitivity demonstrates that the intrinsic shower features that can be used for background suppression and direction reconstruction are still smaller than the pixel sizes of currently operating Cherenkov telescopes.

\subsection{Light Collection Area}

The telescope light collection area determines the signal-to-noise ratio (SNR) of the shower images and 

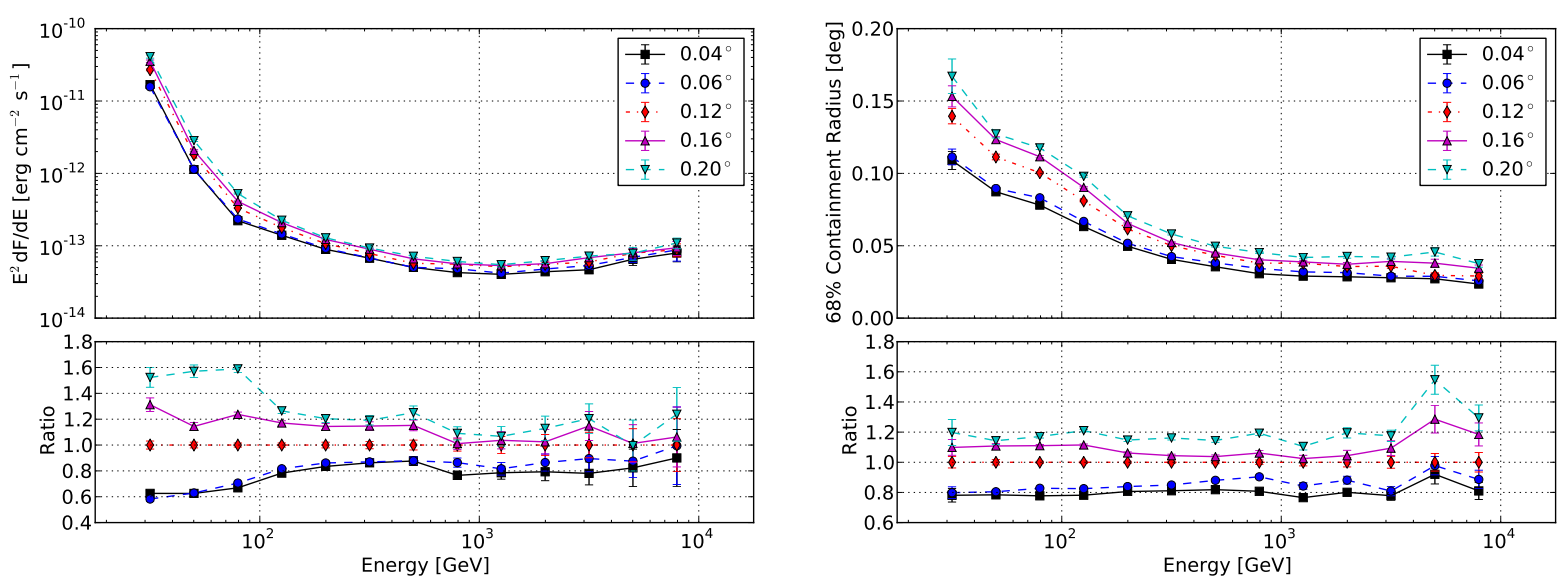

Figure 13: Performance of array M61 simulated with pixel sizes from $0.04^{\circ}$ to $0.20^{\circ}$. Left: Differential point-source sensitivity for a $50 \mathrm{~h}$ observation time. Right: $68 \%$ containment angle of the gamma-ray PSF evaluated after point-source cuts.
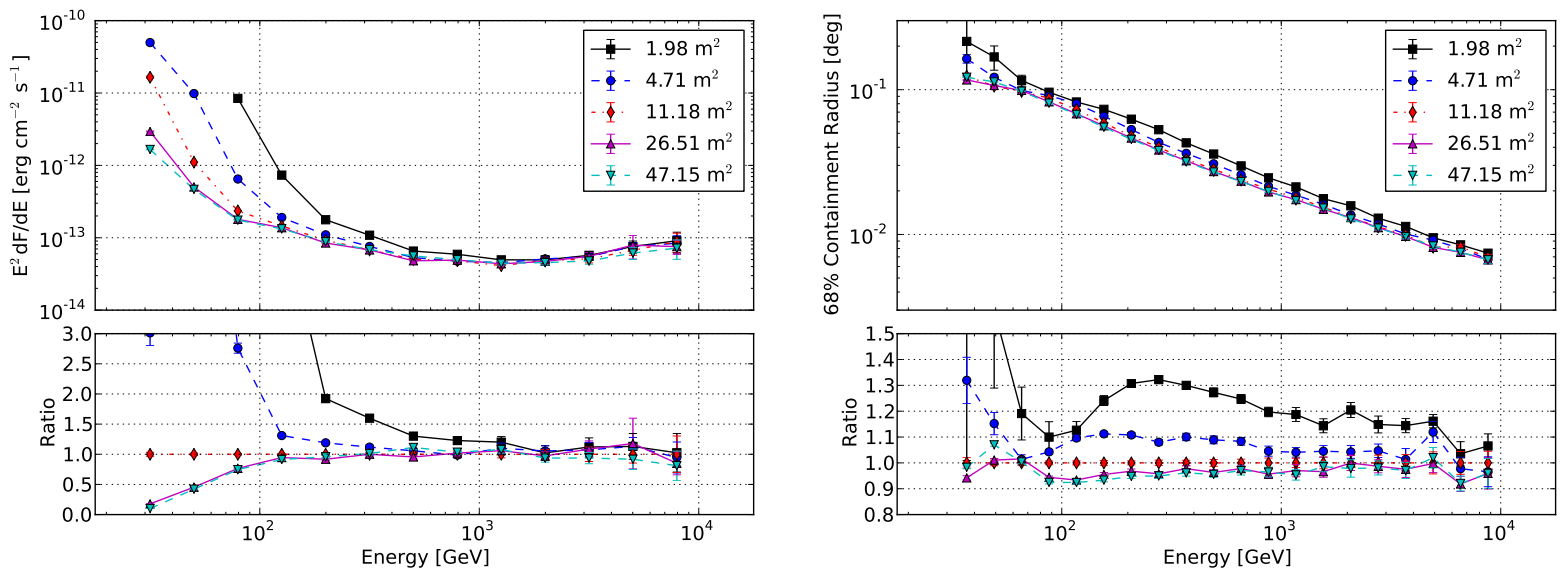

Figure 14: Performance of array M61 simulated with different values of $A_{\text {opt }}: 1.98 \mathrm{~m}^{2}$ (black squares), $4.71 \mathrm{~m}^{2}$ (blue circles), $11.18 \mathrm{~m}^{2}$ (red diamonds), $26.51 \mathrm{~m}^{2}$ (magenta triangles), and $47.15 \mathrm{~m}^{2}$ (cyan triangles). Left: Differential point-source sensitivity for a $50 \mathrm{~h}$ observation time. Right: $68 \%$ containment angle of the gamma-ray PSF after reconstruction cuts.

the efficiency with which these images can be recorded ${ }_{1362}$ by the telescope trigger. Therefore we expect that a 1363 larger $A_{\text {opt }}$ increases the trigger efficiency and provides ${ }_{1364}$ better defined images and hence improves performance 1365 of the array. The role of the $A_{\mathrm{opt}}$ parameter is partic- 1366 ularly relevant for the performance of the array at low energies where the smaller light yield per image makes ${ }^{1367}$ reconstruction and analysis of the gamma-ray showers 1368 more challenging.

The assumed design, size, and cost of the proposed ${ }_{1371}$ telescopes yields distinct $A_{\mathrm{opt}}$ values. We studied the ef- 1372 fect of the $A_{\text {opt }}$ on the gamma-ray PSF and point-source ${ }_{1373}$ sensitivity of the array by examining the performance 1374 of telescope models with $A_{\text {opt }}$ between $2 \mathrm{~m}^{2}$ and $50 \mathrm{~m}^{2} .{ }_{1375}$
These models span the range of light collection areas between SST-like and LST-like telescope designs. The SST, MST, and LST telescope designs have $A_{\text {opt }}$ of approximately $1-2 \mathrm{~m}^{2}, 5-10 \mathrm{~m}^{2}$, and $\sim 50 \mathrm{~m}^{2}$ respectively [46, 33].

Figure 14 shows the comparison of the gamma-ray PSF and point-source sensitivity for telescopes with $A_{\mathrm{opt}}$ between $1.98 \mathrm{~m}^{2}$ (SST-like) and $47.15 \mathrm{~m}^{2}$ (LST-like). $A_{\text {opt }}$ has only a minor effect on the gamma-ray PSF in most of the energy range investigated here. In the middle energy range between $100 \mathrm{GeV}$ and $1 \mathrm{TeV}$ we find an improvement of 5-10\% when increasing the telescope light collection area from $11.18 \mathrm{~m}^{2}$ to $47.15 \mathrm{~m}^{2}$. The almost insignificant improvement around $100 \mathrm{GeV}$ 
is caused by a selection effect of the reconstructed ${ }_{1427}$ gamma-ray events. At these low energies, telescopes ${ }_{1428}$ with smaller $A_{\text {opt }}$ can only trigger on the brightest show- 1429 ers that convert deep in the atmosphere. As discussed in 1430 Section 4.5 the larger interaction depth of these showers ${ }_{1431}$ lessens the impact of the GF and results in a more ac- ${ }_{1432}$ curate reconstruction of the direction. Larger telescopes 1433 can efficiently trigger on showers with both large and ${ }_{1434}$ small interaction depths which results in a larger effec- 1435 tive area but a worsening of the overall gamma-ray PSF. 1436 This effect reverses at the very lowest energies (30- ${ }_{1437}$ $50 \mathrm{GeV}$ ) where the reduced SNR images recorded by 1438 telescopes with small $A_{\text {opt }}$ dominates the reconstruction 1439 quality.

The light collection area has a measurable impact on the point-source sensitivity only at energies below $300 \mathrm{GeV}$ with telescopes with larger light collection area yielding better sensitivity. The increase in sensitivity is most significant below $100 \mathrm{GeV}$ and is a result of the reduction in the telescope trigger threshold and resulting increase in the gamma-ray effective area. The larger light collection area also yields better SNR in the shower images improving the reconstruction of low energy events. At higher energies the impact of light collection area is significantly reduced as the array becomes fully efficient for triggering and reconstructing events that impact within the array boundary. Improving the image SNR provides little improvement at these energies because the reconstruction is predominantly limited by intrinsic shower fluctuations. Remarkably the improvement in point-source sensitivity is almost negligible between telescopes with $26.51 \mathrm{~m}^{2}$ and $47.15 \mathrm{~m}^{2}$ over the whole energy range.

The observed improvements in array performance 1461 above the trigger threshold are small when consider- 1462 ing that light collection area is the dominant parameter ${ }_{1463}$ influencing the telescope cost. Given the small differ- 1464 ences in reconstruction performance, the primary moti- 1465 vation for choosing a telescope design with larger light ${ }_{1466}$ collection area is to reduce the array energy threshold. ${ }_{1467}$ However for an array of fixed cost increasing the light 1468 collection area also entails a reduction in the number of ${ }_{1469}$ telescopes. For gamma-ray energies between $100 \mathrm{GeV}{ }_{1470}$ and $1 \mathrm{TeV}$, a telescope with $A_{\mathrm{opt}}$ of 5-10 $\mathrm{m}^{2}$ (MST-like) ${ }_{1471}$ clearly provides the best performance to cost ratio. Ar- 1472 ray designs that include a small number of telescopes 1473 with larger light collection area can lower the energy 1474 threshold while keeping the cost of the total array within 1475 reasonable limits. Performance of arrays with differ- 1476 ent numbers of telescopes are studied further in Section 1477 4.11

\subsection{Inter-telescope Separation}

The inter-telescope separation determines both the physical area of the array footprint as well as the average number of telescopes that will participate in the reconstruction of individual showers. Smaller telescope separations improve reconstruction quality for contained showers at the cost of lowering the total effective area of the array. Larger telescope separations are generally preferred when optimizing for sensitivity at higher energies since the point-source sensitivity of IACT arrays at moderate exposures (10-50 hours) is signal limited above $1-3 \mathrm{TeV}$. Another important consideration when optimizing the telescope separation is the number of telescopes within the Cherenkov light pool. Telescopes within the Cherenkov light pool sample light emitted by higher energy particles in the shower core and provide a more accurate determination of the shower trajectory. Telescope separations that are comparable to the size of the Cherenkov light pool (100-150 m) ensure that multiple telescopes will sample each shower within its light pool. Finally smaller separations may potentially improve background rejection by increasing the efficiency for detecting Cherenkov light from hadronic subshowers produced in cosmic-ray background events.

The impact of the telescope separation on the gammaray PSF is illustrated in the top panel of Fig. 15 which shows a comparison of arrays with separations between $60 \mathrm{~m}$ and $200 \mathrm{~m}$. In this comparison we consider only showers passing reconstruction cuts with core positions near or within the array boundary. These cuts select events with the best PSF and reduce the differences in performance caused by the finite array size. The reduction of the telescope grid spacing from $120 \mathrm{~m}$ to $60 \mathrm{~m}$ results in a $20 \%$ improvement of the gamma-ray PSF between $30 \mathrm{GeV}$ and $10 \mathrm{TeV}$. However this rather small improvement would require a quadrupling in the number of telescopes to cover a similar area. Thus the improvement of the gamma-ray PSF from reducing the telescope spacing has to be compared to the reduction of effective detector area when fixing the number of available telescopes.

The lower left and right panels of Fig. 15 show the gamma-ray PSF and point-source sensitivity for the set of telescope separations evaluated with a selection optimized for point-source sensitivity. The increase of effective area with larger telescope spacing generally outweighs the reduction of sensitivity due to a worsening of the gamma-ray PSF. The point-source sensitivity improves with increasing telescope spacing at energies above $100 \mathrm{GeV}$ with the best sensitivity achieved with a telescope spacing of 160-200 $\mathrm{m}$. When increasing the telescope spacing to $200 \mathrm{~m}$ a noticeable worsening of 

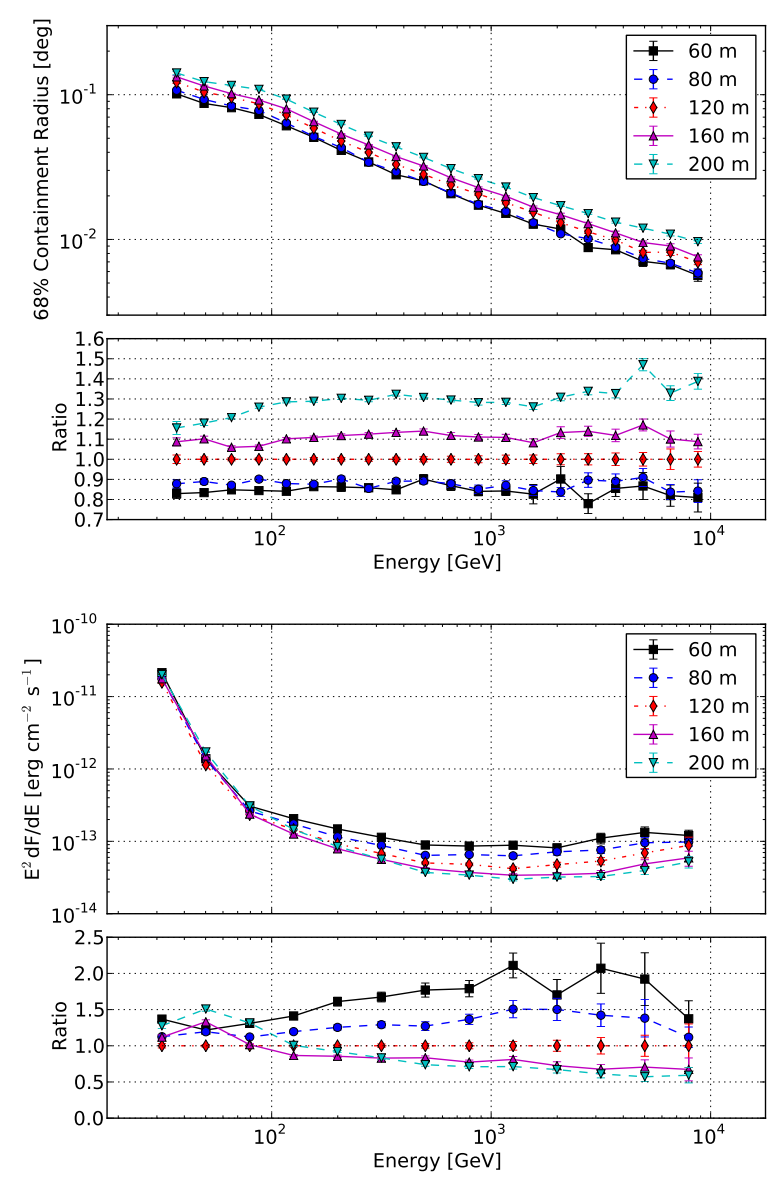
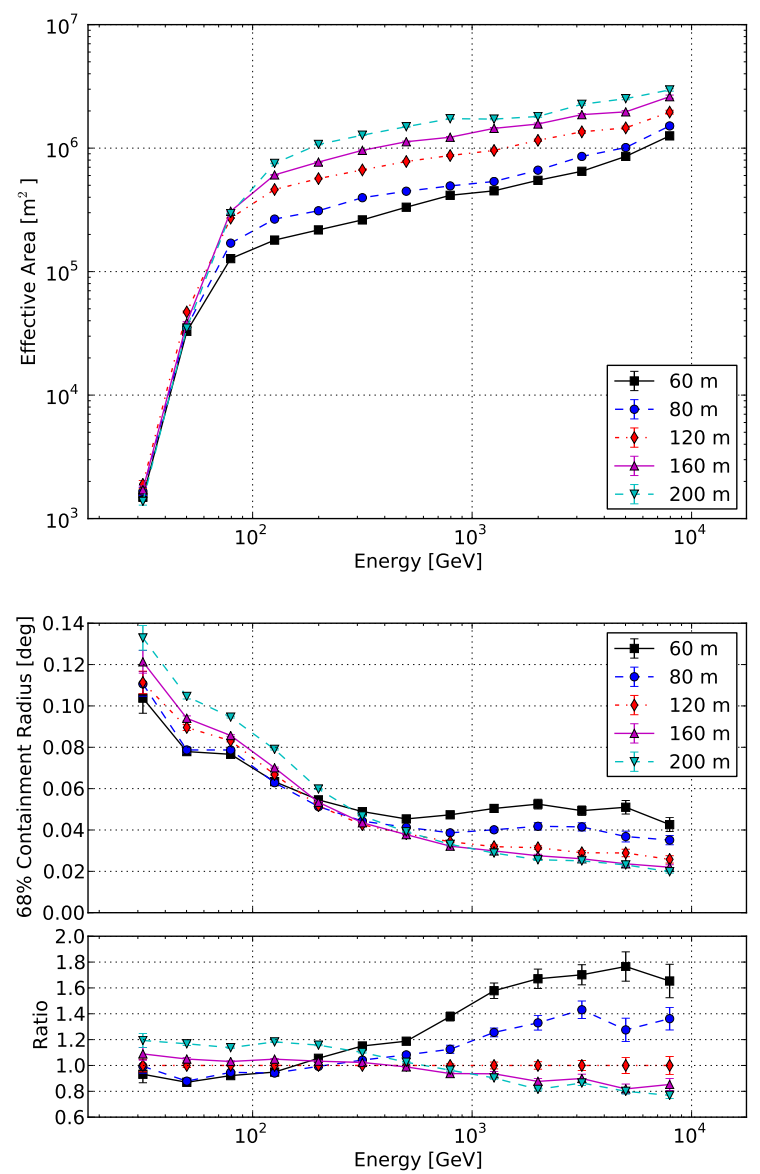

Figure 15: Performance of array M61 simulated with different inter-telescope separations: $60 \mathrm{~m}$ (black squares), $80 \mathrm{~m}$ (blue circles), $120 \mathrm{~m}$ (red diamonds), $160 \mathrm{~m}$ (magenta triangles) and $200 \mathrm{~m}$ (cyan triangles). Top Left: $68 \%$ containment angle of the gamma-ray PSF after reconstruction cuts. Top Right: Gamma-ray effective area after point-source cuts. Bottom Left: Differential point-source sensitivity for a 50 h observation time. Bottom Right: $68 \%$ containment angle of the gamma-ray PSF after point-source cuts.

the sensitivity below $300 \mathrm{GeV}$ is seen because the num- 1496 ber of individual telescopes triggering on each event is ${ }_{1497}$ reduced and hence the information available for direc- 1498 tion and particle type reconstruction.

When evaluated with point-source cuts as shown in the bottom right panel of Fig. 15, the gamma-ray PSF ${ }^{1500}$ above $300 \mathrm{GeV}$ becomes worse as the telescope separa- 1501 tion is decreased. Although a smaller separation gives 1502 a better reconstruction for contained events, the smaller ${ }_{1503}$ array footprint results in a larger fraction of uncontained ${ }_{1504}$ events which tend to dominate the PSF at high energies. 1505 This emphasizes that for most applications where the 1506 maximum sensitivity of the array is required the PSF ${ }_{1507}$ has a quite different behavior compared to the theoret- 1508 ically possible behavior. A wider spacing of the MSTs 1509 will provide a much better performance for most sci- 1510 ence cases compared to a narrow spacing that would be 1511 only beneficial for the very few cases where the gammaray PSF is much more important than sensitivity. Thus the best spacing for the MSTs for all purposes is about $160 \mathrm{~m}$.

\subsection{Trigger Threshold}

The telescope trigger threshold is an important quantity to determine the accessible energy range by any telescope array. The impact of the individual telescope trigger threshold is studied on the differential point-source sensitivity of the M61 baseline array (see Fig. 16. As expected for an MST-like array with $A_{\mathrm{opt}} \simeq$ $10 \mathrm{~m}^{2}$ the trigger threshold has little effect on the sensitivity at energies above $100 \mathrm{GeV}$. At higher energies the telescope trigger becomes fully efficient for showers impacting within the array and reducing the trigger threshold only increases the efficiency for showers on 

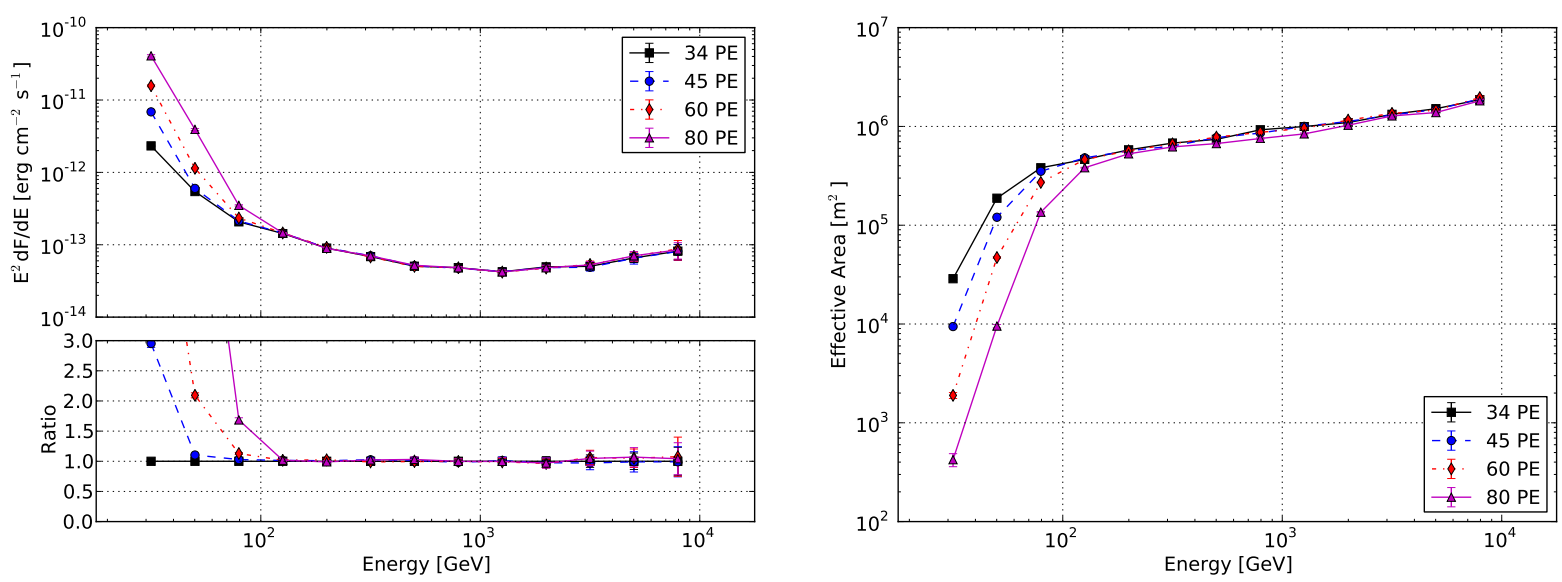

Figure 16: Performance of array M61 simulated with different camera trigger thresholds: 34 PE (black squares), 45 PE (blue circles), 60 PE (red diamonds), 80 PE (magenta triangles). Left: Differential point-source sensitivity for a $50 \mathrm{~h}$ observation time. Right: Gamma-ray effective area after point-source cuts.

the array periphery. Because these distant showers are ${ }_{1542}$ generally not well reconstructed they do not contribute to the array sensitivity.

Reducing the telescope trigger threshold of $\mathrm{Ar}-1547$ ray M61 is found to significantly improve the point- 1548 source sensitivity below $100 \mathrm{GeV}$. A reduction of the 1549 trigger threshold from $80 \mathrm{PE}$ to $34 \mathrm{PE}$ results in a sig- 1550 nificant improvement at energies below $100 \mathrm{GeV}$ and 1551 reaches up to an order of magnitude at $30 \mathrm{GeV}$. How- 1552 ever, in a realistic telescope design the accidental trigger ${ }_{1553}$ rate can not be arbitrarily high due to the limitations on 1554 the readout rate that can be sustained by the telescope 1555 data acquisition. The $60 \mathrm{PE}$ effective trigger threshold 1556 chosen for Array M61 is a realistic target for a trig- ${ }^{1557}$ ger implementation that follows the same design used 1558 by current generation IACTs. Lower trigger thresholds 1559 may be achievable by employing more sophisticated de- 1560 signs for the camera- and array-level triggers such as re- 1561 quiring additional trigger topologies for individual tele- 1562 scopes or higher multiplicities for the array trigger. If 1563 further improvements in the performance of the trigger 1564 can be realized then the presented sensitivities at low en- 1565 ergies could be further improved. Furthermore, it is evi- 1566 dent that the likelihood reconstruction is very efficient at ${ }_{1567}$ low energies and that any reduction in trigger threshold ${ }_{1568}$ is directly translated into an improvement in sensitivity. 1569 The same statement is not necessarily true for the mo- 1570 ment reconstruction that usually has a higher analysis 1571 threshold compared to the likelihood reconstruction as 1572 shown in Fig. 10

\subsection{NSB Rate}

Night-sky background (NSB) is caused by the presence of light sources such as stars, the Moon, and artificial light pollution and represents an irreducible background for the reconstruction and analysis of gammaray air showers. Because the Cherenkov photons detected in a single pixel have an intrinsic arrival time dispersion of 3-6 ns, IACTs can significantly reduce the NSB by integrating the Cherenkov signal in a narrow time window (typically with $\Delta \mathrm{T} \sim 10 \mathrm{~ns}$ ). The integrated NSB level thus depends on both the NSB rate as well as the size of the window used for signal integration. The need for a small integration window motivates camera designs with high bandwidth readout electronics which would allow the integration window to be made as small as possible. The impact of the NSB rate on the sensitivity of the array is also important when considering possible observatory sites and performing observations during moonlight. Moonlight observations can considerably increase the duty cycle of the observatory although the exact amount of observation time gained depends on the NSB rate that the individual telescope can handle.

We studied the impact of NSB on the performance of the array by performing simulations with three NSB flux levels: a baseline flux level with an integral flux of $365 \mathrm{MHz} \mathrm{deg}{ }^{-2} \mathrm{~m}^{-2}$ and NSB fluxes that are 3 and 6 times higher than the baseline flux. As described in Section 2.3 the baseline flux level corresponds to the expected night-sky intensity for a dark, extragalactic field. The higher NSB fluxes are representative of either a higher NSB rate due to operation under high night-sky 

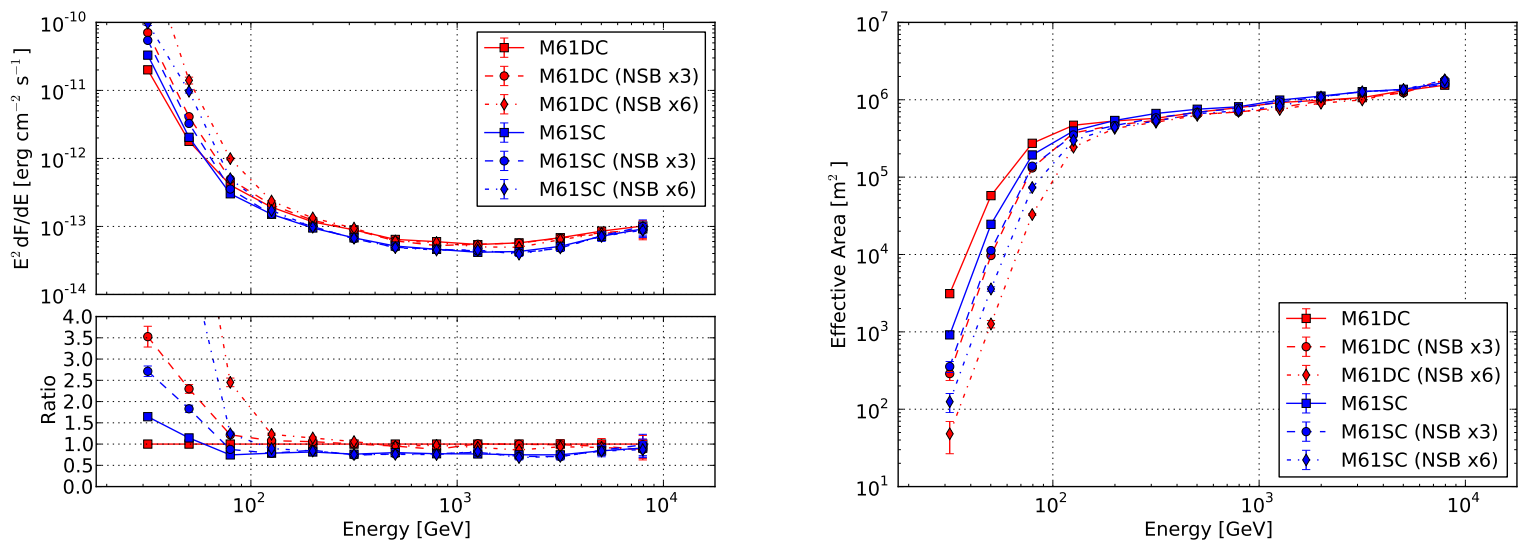

Figure 17: Performance of arrays M61DC (red) and M61SC (blue) simulated with a baseline NSB flux of $365 \mathrm{MHz} \mathrm{deg}^{-2} \mathrm{~m}^{-2}$ (circles and solid lines) and an NSB flux that is 3 (dashed) and 6 (dash-dotted) times higher than the baseline value. Left: Differential point-source sensitivity for a 50 h observation time. Right: Gamma-ray effective area after point-source cuts.

brightness (moonlight) or a longer effective integration 1605 window. A higher NSB rate also increases the rate of 1606 accidental triggers and would require a higher trigger 1607 threshold in order to maintain the accidental trigger rate 1608 at a constant level. For this study we kept the trigger 1609 threshold fixed at its nominal value and only examine 1610 the impact of the NSB on the pixel SNR.

Figure 17 shows the comparison of the point-source ${ }^{161}$ sensitivity and gamma-ray effective area of arrays M61SC and M61DC simulated at the three NSB levels. The NSB level only appreciably affects the sensitivity below $300 \mathrm{GeV}$ where the SNR of the shower image is lowest. Most of the reduction in sensitivity is a result of the lower reconstruction efficiency as low SNR images are removed at the cleaning stage of the analysis. Remarkably the reduction in sensitivity is much more pronounced in the case of larger pixels (DC-like telescope). In case of the SC telescope design, operation at a six times higher NSB rate would only degrade the sensitivity below about $100 \mathrm{GeV}$ and only up to a factor of two. The DC-like design would also suffer sig- ${ }^{1624}$ nificant sensitivity loss only below about $100 \mathrm{GeV}$ but ${ }^{1625}$ to a much greater degree. Here it should be noted that ${ }^{1626}$ the sensitivity advantage of the DC telescopes below $50{ }^{1627}$ $\mathrm{GeV}$ under low NSB is lost in case of three times in- ${ }^{1628}$ creased NSB and that the SC design is better for six ${ }^{1629}$ times higher NSB at all energies.

\subsection{Number of Telescopes in the Array}

One of the most important parameters concerning the ${ }_{1634}$ sensitivity of an IACT array is the number of telescopes. 1635 A larger number of telescopes increases both the total ${ }_{1636}$ effective area for triggering and reconstructing gammaray showers but also increases the average number of telescopes that participate in the reconstruction of each shower. Increasing the number of telescopes leads to better point-source sensitivity and an improved gammaray PSF.

Figure 18 compares the performance of arrays with between 5 and 61 telescopes. We investigate the scaling relation of the improvement in sensitivity with increasing number of telescope. In the limit of an infinite array the point-source sensitivity should scale with the number of telescopes as $\mathrm{N}_{\text {tel }}^{1 / 2}$. However we observe an increase of sensitivity that is slightly better than the $\mathrm{N}_{\text {tel }}^{1 / 2}$ at all energies. This emphasizes that in the case of small telescope arrays increasing the number of telescopes yields larger improvements as compared to the case of extending large arrays. Adding 36 telescopes to a 25 telescope array improves the sensitivity by a factor of $\sim 1.7-1.8$.

In contrast to the point-source sensitivity, the gammaray PSF improves non-uniformly over energy with increasing telescope number. The best improvement is seen at larger energies while at $E<300 \mathrm{GeV}$ the improvement is only clearly visible between 5 and 13 telescopes. At high energies the curves in Fig. 18 show a clearer separation demonstrating that more telescopes help to better localize the showers above $1 \mathrm{TeV}$. The energy dependency has its origin in the fact that only high energy showers produce enough light to trigger distant telescopes. Thus larger arrays with more telescopes benefit at high energies because the average number of telescopes participating in the shower reconstruction is 

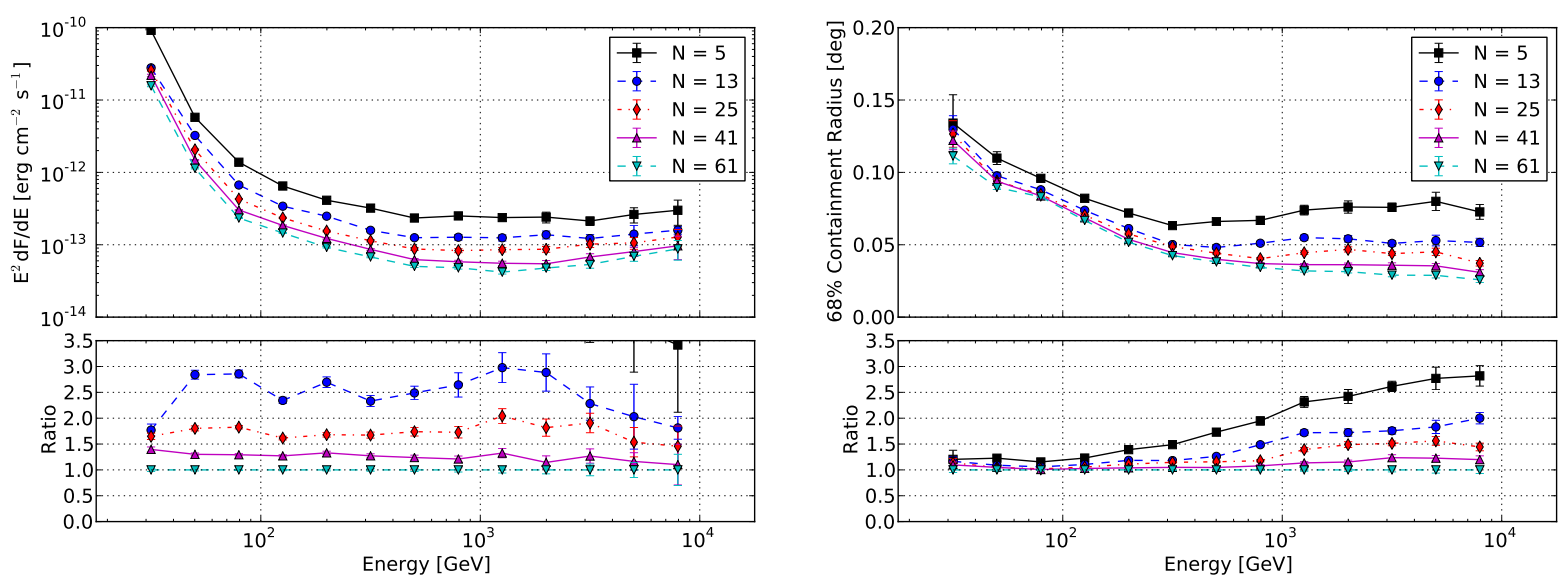

Figure 18: Performance of array layouts with telescope number $\left(\mathrm{N}_{\text {tel }}\right)$ from 5 to 61 . All arrays are simulated with a $120 \mathrm{~m}$ inter-telescope separation and the same telescope model as Array M61. Left: Differential point-source sensitivity for a 50 h observation time. Right: $68 \%$ containment angle of the gamma-ray PSF after applying point-source cuts.

increased. In the case of lower energy showers, the 1668 number of telescopes contributing to the shower anal- 1669 ysis is limited by the telescope spacing and not the ab- 1670 solute number of telescopes in the array. Increasing the 1671 footprint of the array also increases the parallax between 1672 telescopes observing an uncontained shower. The larger ${ }_{1673}$ parallax yields a better shower direction reconstruction 1674 and further improves the reconstruction performance at 1675 high energies.

\subsection{Comparison of Array Designs for CTA}

After studying the effect of individual telescope parameters on the point-source sensitivity and gammaray PSF, we now compare realistic telescope designs against each other to find a suitable array design for CTA. To achieve a comprehensive comparison we investigate all the benchmark arrays defined in Table $2{ }^{1684}$ and give a quantitative comparison between the differ- 1685 ent telescope layouts. Among the benchmark arrays are ${ }^{1686}$ also two more theoretically interesting cases. Array L61 ${ }^{1687}$ is representative of the theoretical limit for an IACT ar- 1688 ray if the budget is not limited and only the number of ${ }^{1689}$ telescopes is fixed. In a similar fashion, Array L5 is ${ }^{1690}$ included to study the contribution of an LST subarray 1691 with 3-5 telescopes such as currently considered for the 1692 baseline configuration of CTA.

Fig. 19 shows that Array M61SC is more sensi- 1694 tive than Array M61DC at all energies above $50 \mathrm{GeV},{ }_{1695}$ where the increase in sensitivity is about $30 \%$. In ad- 1696 dition to the improvement in point-source sensitivity, 1697 the M61SC array also has a better gamma-ray PSF at 1698 all energies. The smaller gamma-ray PSF would help 1699 to determine the morphology of extended sources and help to separate point sources. These additional important effects are difficult to assess quantitatively because they heavily rely on the source population and properties in the sky. The diffuse source is simulated as an uniformly extended disk with a radius of $0.5^{\circ}$. The diffuse-source sensitivity does not show any improvement of the M61SC array over the M61DC array because the gamma-ray PSF does not help to reduce the background but still the M61SC would enable for a nonuniform source to asses the morphology better than Array M61DC. The diffuse source sensitivity emphasizes that the sensitivity gain of the SC array compared to the DC array comes almost entirely from the PSF improvement while the improvement in the background rejection power is marginal.

Array M25DC is representative of the MST subset of the CTA array design as it was planned without a US contribution. Comparing the Array M61SC and Array M61DC to the M25DC baseline configuration, it is obvious that adding MST telescopes will improve the sensitivity of CTA in the key energy range between $100 \mathrm{GeV}$ and about $1 \mathrm{TeV}$ by about a factor two regardless of their design. This is expected from the fact that the sensitivity is improved by the addition of telescopes, as shown in Fig. 18

We also compared the point-source sensitivity of our benchmarks arrays with Array I from [18]. Although the simulations in this paper were performed with different telescope models and a different detector simulation package, this array is representative of the expected performance of the baseline CTA concept. In the 

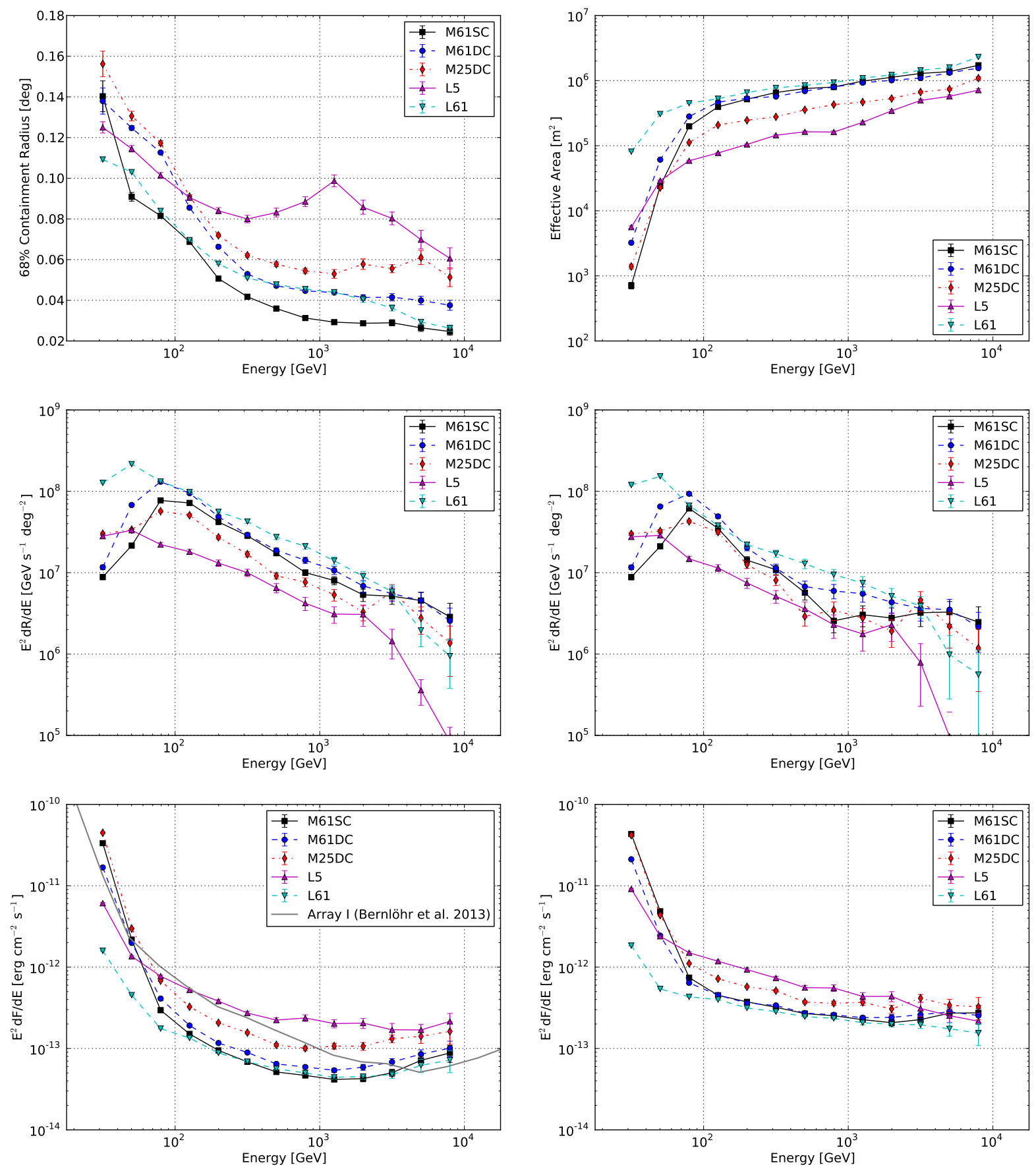

Figure 19: Performance of benchmark arrays: M61SC, M61DC, M25DC, L5, and L61. Top Left: $68 \%$ containment angle of the gamma-ray PSF after applying point-source cuts. Top Right: Gamma-ray effective area after point-source cuts. Middle Left: Differential rate of the total cosmic-ray background (protons and electrons) after point-source cuts. Middle Right: Differential rate of protons after point-source cuts. Bottom Left: Differential point-source sensitivity for a $50 \mathrm{~h}$ observation time. Shown as the dashed black line is the differential sensitivity of Array I from [18] evaluated with the most sensitive analysis at each energy. Bottom Right: Differential diffuse-source sensitivity $\left(D=0.5^{\circ}\right)$ for a $50 \mathrm{~h}$ observation time. 
central energy range from $100 \mathrm{GeV}$ to $3 \mathrm{TeV}$, Arrays 1749 M61DC and M61SC provide a factor of 3-4 improve- 1750 ment in point-source sensitivity relative to Array I. This 1751 improvement can be primarily attributed to the increase 1752 in the number of MSTs from 18 to 61. Array I performs ${ }_{1753}$ better at energies below $50 \mathrm{GeV}$ and above $3 \mathrm{TeV}$ as ${ }_{1754}$ compared to Array M25DC and even Arrays M61DC 1755 and M61SC. This improvement can be attributed to the 1756 inclusion of 56 SSTs and 3 LSTs in Array I. Array L5 ${ }_{1757}$ was simulated with five LSTs very similar to the ones 1758 included in Array I, and the sensitivity curve obtained 1759 for L5 matches very well the sensitivity of Array I at 1760 low energies, demonstrating that the advantage of $\mathrm{Ar}-1761$ ray I at low energies does in fact come from the LSTs. ${ }_{1762}$

Finally Array L61 yielded only an improvement be- 1763 low $100 \mathrm{GeV}$, making such an array impractical based 1764 on the large cost differential between a single MST and 1765 LST. However the performance of this array shows what 1766 is theoretically achievable in the case of no budget con- 1767 straints. Array M61SC provides comparable sensitivity 1768 to Array L61 at all energies above $100 \mathrm{GeV}$ and thus is 1769 very close to the performance of an ideal array in this 1770 energy range.

In case of the diffuse source sensitivity the number 1772 of telescopes is the found to be the most important fac- 1773 tor. Again the addition of MSTs of either type (SC or 1774 DC) would result in a considerable improvement com- 1775 pared to M25DC (similar to Array I) in the whole en- 1776 ergy range. However the improvement is slightly less 1777 significant when compared to the relative improvement 1778 in the point-source sensitivity.

\section{Conclusions}

This paper describes a new simulation and analy- 1784 sis chain that is used to study and compare array and 1785 telescope design concepts for CTA. We specifically fo- 1786 cus on the role of MST arrays which are optimized for ${ }_{1787}$ performance in the core energy range of CTA between 1788 $100 \mathrm{GeV}$ and $1 \mathrm{TeV}$. The simplified detector model used 1789 for this study allows for investigation of a wide range 1790 of telescope parameters: effective light collection area, 1791 optical PSF, camera pixel size, effective camera trig- 1792 ger threshold, and effective integration window in time. 1793 The simplified telescope description allows us to isolate 1794 the most important telescope design characteristics and 1795 fully explore their influence on the performance of the 1796 full array. Realistic telescope designs can be mapped to 1797 our simplified detector model by choosing telescope pa- 1798 rameters that are matched to the physical characteristics 1799 of each design (mirror area, focal length, photosensor 1800 efficiency, etc.). This paper also investigates several aspects of the array geometry optimization including the impact of the number of telescopes and their separation on array performance.

A benchmark telescope array was used to assess the influence of each of the telescope and array parameters. Performance is evaluated for nominal observing conditions corresponding to a zenith angle of $20^{\circ}$ and an NSB rate computed for a dark extragalatic field. We also examined the influence of the GF and higher NSB rates. Under all conditions, an optimized analysis is performed using a likelihood reconstruction based on simulated image templates and BDTs for signal extraction.

The likelihood reconstruction based on simulated templates offers a factor of two improvement in point source sensitivity (30-40\% improvement in gamma-ray PSF), as well as a reduced energy threshold relative to image moment-based analysis. The likelihood reconstruction takes advantage of the possibility of fully resolving showers with a finely pixelated camera. This technique, coupled with BDTs for event selection, allowed us to compare arrays very close to their maximum achievable sensitivity.

We find that the substantial improvements in both the gamma-ray point-source sensitivity and angular resolution of an IACT array can be realized by telescopes with imaging resolution better than current-generation IACT designs. We find a 30-40\% improvement in the gamma-ray point-source sensitivity between $100 \mathrm{GeV}$ and $3 \mathrm{TeV}$ when the telescope pixel size is reduced from $0.16^{\circ}$ to $0.06^{\circ}$. The gain in point-source sensitivity comes primarily from the improvement in the gammaray angular reconstruction enabled by the higher resolution imaging of the shower axis. Over the same energy range, the performance of an MST array is much less sensitive to the telescope light collection area and trigger threshold. We find that these parameters are important in determining the array energy threshold but have little influence on the array performance above the threshold energy.

With higher resolution shower images, the GF becomes more relevant than ever for the sensitivity of an IACT array. To determine the impact of the GF, we compared the same array simulated with values of $B_{\perp}$ between $0 \mu \mathrm{T}$ and $20.7 \mu \mathrm{T}$. For an MST array, the impact of the GF is largest around $100 \mathrm{GeV}$ where the point-source sensitivity is reduced by $50 \%$. The GF should be an important factor in selecting a site for future arrays and possibly for designing an observing strategy.

Increasing the number of telescopes in the array expands the effective area, improves reconstruction, and 
increases background rejection capabilities. The sensi- 1850 tivity can be improved faster at very low and very high energies by adding LSTs and SSTs. However, in the ${ }^{1851}$ energy range between a few hundred $\mathrm{GeV}$ and about ${ }^{1852}$ ten TeV, expanding the MST array efficiently improves ${ }^{1}$ the sensitivity, regardless of the telescope design. In the limit of a finite array for which uncontained showers constitute a significant fraction of the total reconstructed 1855 event sample, the improvement in point-source sensitivity scales faster than the square root of the number of ${ }_{1857} 856$ telescopes between $300 \mathrm{GeV}$ and $3 \mathrm{TeV}$. If the baseline ${ }^{1858}$ CTA design is expanded to include 36 more MSTs, the ${ }^{1859}$ point-source sensitivity in the core energy is improved ${ }_{1866}{ }_{1860}$ by a factor of two.

When considering arrays with the same number of ${ }^{1864}$ telescopes, we find that the SC telescope design yields ${ }_{1866} 865$ a 30-40\% improvement in point-source sensitivity over 1867 the DC telescope design because of its superior imag- ${ }^{1868}$ ing resolution. The DC telescope on the other hand ${ }_{1870}^{1869}$ has a slightly lower energy threshold resulting in better ${ }_{1871}$ point source sensitivity below $75 \mathrm{GeV}$. The improved 1872 performance in a wide energy range from the SC design ${ }^{1873}$ warrants further investigation. The improved sensitiv- ${ }_{1875} 874$ ity reduces the total exposure time required for every ${ }_{1876}$ science topic, while the smaller gamma-ray PSF addi- 1877 tionally helps with source confusion and morphology ${ }^{1878}$ studies. The higher resolution shower images of the SC- ${ }_{1890} 1879$ like telescopes are also much less affected by noise from ${ }_{1881}$ NSB. This translates to a much lower energy thresh- 1882 old during brighter sky conditions, e.g. in the galactic ${ }^{1883}$ plane. This may lead to a much higher effective duty ${ }_{1885} 1884$ cycle since observations can be continued into brighter ${ }_{1886}$ moon phases without sacrificing the low energy regime. ${ }^{1887}$

While the SC-like array is more sensitive in compari- ${ }^{1889}$ son to the DC-like design, no SC MST telescope has yet 1891 been built. Construction of an SC prototype at the site of ${ }^{1892}$ VERITAS is under way. This prototype offers a chance ${ }^{1893}$ to study the performance of the SC optics in realistic ${ }_{1895} 894$ circumstances. This experience should also provide a 1896 more realistic cost model for the two-mirror systems.

At this point in the design of CTA, it is unlikely that ${ }_{1900}$ all MST telescopes would be of the SC design. If the SC 1901 prototype can be built successfully and cost-efficiently, ${ }_{1902}$ the baseline CTA array could be expanded to include an ${ }_{1904} 1903$ additional number of SC MSTs. The study of mixed ar- 1905 rays is ongoing. No matter which optical design is cho- 1906 sen, expanding the MST arrays offers significant ben- ${ }^{1907}$ efits for the performance of CTA in the central energy ${ }_{1909}^{1908}$ range between $100 \mathrm{GeV}$ and $1 \mathrm{TeV}$.

\section{Acknowledgements}

We thank Brian Humensky, Emiliano Carmona, and the anonymous referees for their valuable comments. This work was supported in part by the Department of Energy contract DE-AC02-76SF00515.

\section{References}

[1] Bell, A.R. Cosmic ray acceleration. Astroparticle Physics, 43(0):56 - 70, 2013. ISSN 0927-6505.

[2] Acero, F., Bamba, A., Casanova, S., et al. Gamma-ray signatures of cosmic ray acceleration, propagation, and confinement in the era of CTA. Astroparticle Physics, 43:276-286, March 2013. 1209.0582

[3] Halzen, Francis. Pionic photons and neutrinos from cosmic ray accelerators. Astroparticle Physics, 43(0):155 - 162, 2013. ISSN 0927-6505.

[4] Doro, M. et al. Dark matter and fundamental physics with the cherenkov telescope array. Astroparticle Physics, 43(0):189 214, 2013. ISSN 0927-6505.

[5] Bergström, L. Dark matter and imaging air Cherenkov arrays. Astroparticle Physics, 43:44-49, March 2013.

[6] Ellis, John and Mavromatos, Nick E. Probes of lorentz violation. Astroparticle Physics, 43(0):50 - 55, 2013. ISSN 0927-6505.

[7] de Oña-Wilhelmi, E., Rudak, B., Barrio, J. A., et al. Prospects for observations of pulsars and pulsar wind nebulae with CTA. Astroparticle Physics, 43:287-300, March 2013. 1209.0357

[8] Paredes, J.M. et al. Binaries with the eyes of CTA. Astroparticle Physics, 43(0):301 - 316, 2013. ISSN 0927-6505.

[9] Aharonian, Felix A. Gamma rays from supernova remnants. Astroparticle Physics, 43(0):71 - 80, 2013. ISSN 0927-6505.

[10] Reimer, A. and Böttcher, M. Studies of active galactic nuclei with CTA. Astroparticle Physics, 43:103-111, March 2013. 1208.5926

[11] Sol, H. et al. Active galactic nuclei under the scrutiny of CTA. Astroparticle Physics, 43(0):215 - 240, 2013. ISSN 0927-6505.

[12] Inoue, S., Granot, J., O'Brien, P. T., et al. Gamma-ray burst science in the era of the Cherenkov Telescope Array. Astroparticle Physics, 43:252-275, March 2013. 1301.3014

[13] Mészáros, P. Gamma ray bursts. Astroparticle Physics, 43:134 141, March 2013. 1204.1897

[14] Domínguez, A. and Prada, F. Measurement of the Expansion Rate of the Universe from $\gamma$-Ray Attenuation. ApJ, 771:L34, July 2013. 1305.2163

[15] Davies, J. M. and Cotton, E. S. Design of the Quartermaster Solar Furnace. Solar Energy Sci. Eng., 1:16 - 22, 1957.

[16] Lewis, D. A. Optical characteristics of the Whipple Observatory TeV gamma-ray imaging telescope. Exp. Astron., 1:213 - 236, 1990.

[17] Bernlohr, Konrad. Simulation of Imaging Atmospheric Cherenkov Telescopes with CORSIKA and sim_telarray. Astropart.Phys., 30:149-158, 2008. 0808.2253

[18] Bernlöhr, K., Barnacka, A., Becherini, Y., et al. Monte Carlo design studies for the Cherenkov Telescope Array. Astroparticle Physics, 43:171-188, March 2013. 1210.3503

[19] Vassiliev, Vladimir, Brousseau, P.F., and Fegan, S.J. Wide field Ritchey-Chretien telescope for ground-based gamma-ray astronomy. Astropart.Phys., 28:10-27, 2007. astro-ph/ 0612718

[20] Vassiliev, V. V. and Fegan, S. J. Schwarzschild-Couder twomirror telescope for ground-based $\gamma$-ray astronomy. International Cosmic Ray Conference, 3:1445-1448, 2008. 0708. 2741 
[21] Rousselle, Julien et al. Schwarzschild-Couder telescope for the 1976 Cherenkov Telescope Array: Development of the Optical Sys- 1977 tem. 2013. 1307.4072

[22] Aharonian, F. et al. Observations of the Crab Nebula with 1979 H.E.S.S. Astron.Astrophys., 457:899-915, 2006. astro-ph/ 1980 0607333

[23] MAGIC: https://wwwmagic.mpp.mpg.de/

[24] VERITAS: http://veritas.sao.arizona.edu/ the Cherenkov Telescope Array CTA: an advanced facility for 1985 ground-based high-energy gamma-ray astronomy. Experimen- 1986 tal Astronomy, 32:193-316, December 2011. 1008.3703 1987

[26] Heck, D., Knapp, J., Capdevielle, J. N., Schatz, G., and Thouw, 1988 T. CORSIKA: a Monte Carlo code to simulate extensive air 1989 showers. February 1998.

27] Ostapchenko, S. QGSJET-II: towards reliable description of 1991 very high energy hadronic interactions. Nuclear Physics B Pro- 1992 ceedings Supplements, 151:143-146, January 2006. arXiv: 1993 hep-ph/0412332

[28] Bernlöhr, K. Simulation of imaging atmospheric Cherenkov telescopes with CORSIKA and sim_telarray. Astroparticle Physics, 30:149-158, October 2008. 0808.2253

[29] Kneizys, F. X. and et al. The MODTRAN 2/3 report and LOWTRAN 7 model, Tech. rep., Phillips Laboratory. 1996.

[30] Benn, C. R. and Ellison, S. L. Brightness of the night sky over La Palma. New A Rev., 42:503-507, November 1998. arXiv: astro-ph/9909153

[31] Funk, S., Hermann, G., Hinton, J., et al. The trigger system of the H.E.S.S. telescope array. Astroparticle Physics, 22:285-296, November 2004. astro-ph/0408375

[32] Holder, J., Atkins, R. W., Badran, H. M., et al. The first VERITAS telescope. Astroparticle Physics, 25:391-401, July 2006. astro-ph/0604119

[33] Bernlöhr, K., Barnacka, A., Becherini, Y., et al. Progress in Monte Carlo design and optimization of the Cherenkov Telescope Array. eprint arXiv:1307.2773, July 2013. 1307.2773

[34] Daum, A., Hermann, G., Heß, M., et al. First results on the performance of the HEGRA IACT array. Astroparticle Physics, 8:1-11, December 1997.

[35] Hillas, A. M. Cerenkov light images of EAS produced by primary gamma. In F. C. Jones, editor, International Cosmic Ray Conference, pages 445-448. August 1985.

[36] de Naurois, M. and Rolland, L. A high performance likelihood reconstruction of $\gamma$-rays for imaging atmospheric Cherenkov telescopes. Astroparticle Physics, 32:231-252, December 2009. 0907.2610

[37] Krawczynski, H., Carter-Lewis, D. A., Duke, C., et al. Gamma hadron separation methods for the VERITAS array of four imaging atmospheric Cherenkov telescopes. Astroparticle Physics, 25:380-390, July 2006. arXiv:astro-ph/0604508

[38] Hoecker, A., Speckmayer, P., Stelzer, J., et al. TMVA - Toolkit for Multivariate Data Analysis. ArXiv Physics e-prints, March 2007. arXiv: physics/0703039

[39] Ohm, S., van Eldik, C., and Egberts, K. $\gamma$ /hadron separation in very-high-energy $\gamma$-ray astronomy using a multivariate analysis method. Astroparticle Physics, 31:383-391, June 2009. 0904. 1136

[40] Li, T.-P. and Ma, Y.-Q. Analysis methods for results in gammaray astronomy. ApJ, 272:317-324, September 1983.

[41] Lemoine-Goumard, M., Degrange, B., and Tluczykont, M. Selection and 3D-reconstruction of gamma-ray-induced air showers with a stereoscopic system of atmospheric Cherenkov telescopes. Astroparticle Physics, 25:195-211, April 2006. astro-ph/0601373

[42] Bowden, C. C. G., Bradbury, S. M., Chadwick, P. M., et al. The effect of the geomagnetic field on $\mathrm{TeV}$ gamma -ray detection. Journal of Physics G Nuclear Physics, 18:L55-L60, February 1992.

[43] Chadwick, P. M., Lyons, K., McComb, T. J. L., et al. Geomagnetic effects on atmospheric Cerenkov images. Journal of Physics G Nuclear Physics, 25:1223-1233, June 1999. astro-ph/9904082

[44] Commichau, S. C., Biland, A., Contreras, J. L., et al. Monte Carlo studies of geomagnetic field effects on the imaging air Cherenkov technique for the MAGIC telescope site. Nuclear Instruments and Methods in Physics Research A, 595:572-586, October 2008. 0802.2551

[45] Szanecki, M., Bernlöhr, K., Sobczyńska, D., et al. Influence of the geomagnetic field on the IACT detection technique for possible sites of CTA observatories. Astroparticle Physics, 45:1-12, May 2013. 1302.6387

[46] Pareschi, G., Agnetta, G., Antonelli, L. A., et al. The dualmirror Small Size Telescope for the Cherenkov Telescope Array. ArXiv e-prints, July 2013. 1307.4962 